\title{
Soft theorems in maximally supersymmetric theories
}

\author{
Zheng-Wen Liu ${ }^{\mathrm{a}}$ \\ Department of Physics, Renmin University of China, Beijing 100872, People's Republic of China
}

Received: 15 December 2014 / Accepted: 3 February 2015 / Published online: 4 March 2015

(C) The Author(s) 2015. This article is published with open access at Springerlink.com

\begin{abstract}
In this paper we study the supersymmetric generalization of the new soft theorem which was proposed by Cachazo and Strominger recently. At tree level, we prove the validity of the super soft theorems in both $\mathcal{N}=4$ superYang-Mills theory and $\mathcal{N}=8$ supergravity using super$\mathrm{BCFW}$ recursion relations. We verify these theorems exactly by showing some examples.
\end{abstract}

\section{Contents}

1 Introduction . . . . . . . . . . . . 1

2 New soft graviton theorem and soft gluon theorem . 2

2.1 Cachazo-Strominger's new soft graviton theorem 2

2.2 Soft gluon theorem in Yang-Mills theory . . . . 3

2.3 Gravity soft operators as double copy of YangMills soft operators . . . . . . . . . . . . 3

3 Soft theorem in $\mathcal{N}=4$ super-Yang-Mills theory . . 4

3.1 Super soft theorem in $\mathcal{N}=4 \mathrm{SYM} \ldots \ldots \ldots 5$

3.2 MHV examples . . . . . . . . . . . . . 6

3.3 Six-point NMHV examples . . . . . . . . . 7

4 Super soft theorem in $\mathcal{N}=8$ supergravity . . . . . 8

4.1 Super soft theorem in $\mathcal{N}=8$ SUGRA . . . . 8

4.2 MHV examples ............ . . . . . . . . . 9

4.2.1 Soft gravitino . . . . . . . . . . . 10

4.2.2 Soft gravi-photon . . . . . . . . . . . . 10

4.2.3 Soft gravi-photino and soft scalar . . . . 12

4.3 SUGRA soft operators as double copy of SYM soft operators . . . . . . . . . . . 12

5 Conclusion and discussions . . . . . . . . . 13

Appendix A: Alternative derivation of soft theorem

in $\mathcal{N}=4 \mathrm{SYM} \ldots \ldots \ldots \ldots$

Appendix B: Sub-sub-leading soft operator in $\mathcal{N}=8$

SUGRA . . . . . . . . . . . . 15

References ............... . 17

a e-mail: zhengwen@ruc.edu.cn

\section{Introduction}

Over the past few decades, there was great progress on the understanding of physical and mathematical structures of the perturbative scattering amplitudes in gauge and gravity theories. Among these remarkable developments, a recent advance is the soft behavior of scattering amplitudes when one or more external legs tend to zero in both gravity and Yang-Mills [1-27].

The study on soft behavior of the amplitudes (in gravity) goes back to Steven Weinberg who proposed the universal leading soft-graviton behavior using the Feynman diagrams technique more than 50 years ago [28]. The leading softgraviton behavior of the amplitudes, also called "Weinberg's theorem", is uncorrected to all loop orders. At sub-leading order, the soft photon behavior and the soft graviton behavior were also studied by using Feynman diagrams [29-31].

Very recently, sub-leading and sub-sub-leading softgraviton divergences of amplitudes were proposed [32] beyond Weinberg's theorem. In [32], Cachazo and Strominger presented a proof for tree-level amplitudes of gravitons using BCFW recursion relations [33-38] in the spinorhelicity formulism [39-41]. Similarly, the sub-leading soft divergence for Yang-Mills amplitudes was obtained by same analysis in [1]. As pointed out in [4], the sub-leading divergence is vanishing in pure Yang-Mills amplitudes. However, we will see that the sub-leading Yang-Mills soft operator is necessary for "KLT-construction" of gravity soft operators in this paper.

On the other hand, a lot of remarkable progress has also been made on the understanding of the soft theorem from the viewpoint of symmetry principles.

The leading and sub-leading soft graviton theorems are understood as Ward identities of BMS symmetry [25-27]. The leading [18] and sub-leading [17] soft photon theorems were interpreted as asymptotic symmetries of $\mathcal{S}$-matrix in massless QED. 
Now aspects of soft theorems have been investigated along many different directions [1-27]. Loop corrections of soft theorems were investigated in both gravity and Yang-Mills [2-5]. Various different methods were used to derive the soft theorems, including Feynman diagram approach [8] and conformal symmetry approach [16] for Yang-Mills, the Poincaré symmetry and gauge symmetry approach [6,7], ambitwistor string approach [15] for Yang-Mills and gravity. The new soft graviton theorem was also obtained from the soft gluon theorem via the Kawai-Lewellen-Tye (KLT) relations [19]. The scattering equations [42-45], or Cachazo-He-Yuan (CHY) formulae, were used to study the soft theorems in arbitrary number of dimensions. The soft divergence in string theory was also investigated in $[4,14]$.

It is natural and interesting to investigate soft divergences of amplitudes in supersymmetric theories. In this paper, we mainly focus on the soft theorems in the maximally supersymmetric theories, i.e., $\mathcal{N}=4$ super-Yang-Mills (SYM) theory and $\mathcal{N}=8$ supergravity (SUGRA), in 4 spacetime dimensions. Great progress on analytical calculations of the scattering amplitudes, in particular at tree-level, has been achieved in $\mathcal{N}=4$ SYM and $\mathcal{N}=8$ SUGRA. A lot of remarkable and interesting structures of scattering amplitudes were discovered [46-52]. Many novel methods for perturbative scattering amplitudes were proposed, and a large number of amplitudes were also analytically computed in various theories [53-68]. Scattering amplitudes in massless QCD were also studied in $[69,70]$.

For example, by solving super-BCFW recursion relation [33-35,56], the compact analytical formulae for all tree amplitudes were presented in $\mathcal{N}=4$ SYM [57], also in massless QCD with up to four quark-anti-quark pairs [61].

These previous works provide a solid foundation for our study.

We will systematically study the soft theorems in $\mathcal{N}=4$ SYM and $\mathcal{N}=8$ SUGRA using super-BCFW recursion relations in this work. We will pay special attention to soft gravitino divergence, soft gravi-photon divergence for SUGRA and soft gluino divergence for SYM.

This paper is organized as follows. In the next section, we briefly review soft theorems in both gravity and Yang-Mills. In Sect. 3, we present the super soft theorem in $\mathcal{N}=4$ superYang-Mills with a rigorous proof in detail. There we also provide some lower-point amplitudes to test the validity of the super soft theorem, especially for soft gluino divergence.

In Sect. 4, we study the super soft theorem in $\mathcal{N}=8$ supergravity in detail. As examples, we show that the super soft theorem is consistent with SUSY Ward identity in the MHV sector. Several four-boson amplitudes are also examined exactly.

In Sect. 5, we conclude this paper with some brief discussions. Appendix A provides an alternative derivation of soft theorem in $\mathcal{N}=4$ SYM. Appendix B gives calcula- tional details of the sub-sub-leading soft operator in $\mathcal{N}=8$ SUGRA.

\section{New soft graviton theorem and soft gluon theorem}

In this section we briefly review the new soft graviton theorem [32] and the soft gluon theorem [1,71]. We also show that each order soft operator in gravity may be expressed as a double copy of Yang-Mills soft operators with gauge freedom.

\subsection{Cachazo-Strominger's new soft graviton theorem}

An on-shell $(n+1)$-point scattering amplitude including an external graviton with momentum $k_{s}$ may be denoted

$\mathcal{M}_{n+1}=\mathcal{M}_{n+1}\left(k_{1}, \ldots, k_{n}, k_{s}\right)$.

In the soft limit $k_{s} \rightarrow 0$, the amplitude $\mathcal{M}_{n+1}$ behaves as

$$
\begin{aligned}
& \mathcal{M}_{n+1}\left(k_{1}, \ldots, k_{n}, k_{s}\right) \\
& \quad=\left(S^{(0)}+S^{(1)}+S^{(2)}\right) \mathcal{M}_{n}\left(k_{1}, \ldots, k_{n}\right)+\mathcal{O}\left(k_{s}^{2}\right) .
\end{aligned}
$$

The soft operators are given by

$$
\begin{aligned}
S^{(0)} & =\sum_{a=1}^{n} \frac{E_{\mu \nu} k_{a}^{\mu} k_{a}^{\nu}}{k_{s} \cdot k_{a}}, \\
S^{(1)} & =-i \sum_{a=1}^{n} \frac{E_{\mu \nu} k_{a}^{\mu}\left(k_{s \sigma} J_{a}^{\sigma \nu}\right)}{k_{s} \cdot k_{a}}, \\
S^{(2)} & =-\frac{1}{2} \sum_{a=1}^{n} \frac{E_{\mu \nu}\left(k_{s \rho} J_{a}^{\rho \mu}\right)\left(k_{s \sigma} J_{a}^{\sigma \nu}\right)}{k_{s} \cdot k_{a}},
\end{aligned}
$$

where $E_{\mu \nu}$ is the polarization tensor of the soft graviton $s$ and $J_{a}^{\mu \nu}$ is the total angular momentum of the $a$ th external leg. It is easy to check that all the soft operators are gauge invariant [32]. The leading soft factor $S^{(0)}$ proposed by Steven Weinberg is uncorrected by all loop orders [28] while sub-leading and sub-sub-leading soft operators are not, as discussed in [2-6].

In the spinor-helicity formulism, the momentum vector $k^{\mu}$ of an on-shell massless particle may be represented as a bispinor, i.e.,

$k_{\alpha \dot{\alpha}}=k_{\mu} \sigma_{\alpha \dot{\alpha}}^{\mu}=\lambda_{\alpha} \tilde{\lambda}_{\dot{\alpha}}$.

Introducing an infinitesimal soft parameter $\epsilon$, one can write soft limit of the momentum $k_{s}$ of soft particle as

$$
\begin{aligned}
k_{s} & \rightarrow \epsilon k_{s}=\epsilon \lambda_{s} \tilde{\lambda}_{s} \\
& \Longrightarrow \lambda_{s} \rightarrow \epsilon^{\delta} \lambda_{s}, \quad \tilde{\lambda}_{s} \rightarrow \epsilon^{1-\delta} \tilde{\lambda}_{s} .
\end{aligned}
$$


Here different choices of $\delta$ in physical amplitudes can be linked to each other via the little group transformation, i.e.,

$\mathcal{M}\left(\left\{t \lambda_{s}, t^{-1} \tilde{\lambda}_{s}, h_{s}\right\}\right)=t^{-2 h_{s}} \mathcal{M}\left(\left\{\lambda_{s}, \tilde{\lambda}_{s}, h_{s}\right\}\right)$,

where $h_{s}$ is helicity of the particle $s$. In this paper, one employs the holomorphic soft limit [56]:

$\lambda_{s} \rightarrow \epsilon \lambda_{s}$

in which only the holomorphic spinor $\lambda_{s}$ tends to zero while the anti-holomorphic spinor $\tilde{\lambda}_{s}$ remains unchangeable. The new soft graviton theorem (2.2) is then

$$
\begin{aligned}
& \mathcal{M}_{n+1}\left(\ldots,\left\{\epsilon \lambda_{s}, \tilde{\lambda}_{s}\right\}\right) \\
& \quad=\left(\frac{1}{\epsilon^{3}} S^{(0)}+\frac{1}{\epsilon^{2}} S^{(1)}+\frac{1}{\epsilon} S^{(2)}\right) \mathcal{M}_{n}+\mathcal{O}\left(\epsilon^{0}\right) .
\end{aligned}
$$

In the spinor-helicity formulism, ${ }^{1}$ the soft operators are given by

$$
\begin{aligned}
& S^{(0)}=\sum_{a=1}^{n} \frac{[s, a]\langle x, a\rangle\langle y, a\rangle}{\langle s, a\rangle\langle x, s\rangle\langle y, s\rangle}, \\
& S^{(1)}=\frac{1}{2} \sum_{a=1}^{n} \frac{[s, a]}{\langle s, a\rangle}\left(\frac{\langle x, a\rangle}{\langle x, s\rangle}+\frac{\langle y, a\rangle}{\langle y, s\rangle}\right) \tilde{\lambda}_{s \dot{\alpha}} \frac{\partial}{\partial \tilde{\lambda}_{a \dot{\alpha}}}, \\
& S^{(2)}=\frac{1}{2} \sum_{a=1}^{n} \frac{[s, a]}{\langle s, a\rangle} \tilde{\lambda}_{s \dot{\alpha}} \tilde{\lambda}_{s \dot{\beta}} \frac{\partial^{2}}{\partial \tilde{\lambda}_{a \dot{\alpha}} \partial \tilde{\lambda}_{a \dot{\beta}}} .
\end{aligned}
$$

Here one has assigned soft graviton the helicity $h_{s}=+2$, just a convention. Spinors $\lambda_{x}, \lambda_{y}$ are two arbitrary choosen reference spinors and the freedom in this choice is equivalent to the gauge freedom.

\subsection{Soft gluon theorem in Yang-Mills theory}

The similar soft behavior of the scattering amplitudes appears also in Yang-Mills theory $[1,71]$. In the soft limit of the momentum of a gluon, $k_{s} \rightarrow 0$, an on-shell color-ordered Yang-Mills amplitude $\mathcal{A}_{n+1}$ becomes

$$
\begin{aligned}
& \mathcal{A}_{n+1}\left(k_{1}, \ldots, k_{n}, k_{s}\right) \\
& \quad=\left(S_{\mathrm{YM}}^{(0)}+S_{\mathrm{YM}}^{(1)}\right) \mathcal{A}_{n}\left(k_{1}, \ldots, k_{n}\right)+\mathcal{O}\left(k_{s}\right),
\end{aligned}
$$

where the leading soft (eikonal) factor [71] is

\footnotetext{
${ }^{1}$ In this paper, we mainly follow the notation of ref. [32]. The spinor products are defined as $\langle i, j\rangle=\epsilon^{\alpha \beta} \lambda_{i \alpha} \lambda_{j \beta}=\lambda_{i \alpha} \lambda_{j}^{\alpha}$ and $[i, j]=$ $\epsilon^{\dot{\alpha} \dot{\beta}} \tilde{\lambda}_{i \dot{\alpha}} \tilde{\lambda}_{j \dot{\beta}}=\tilde{\lambda}_{i \dot{\alpha}} \tilde{\lambda}_{j}^{\dot{\alpha}}$, and we use the convention $s_{i j}=\langle i, j\rangle[i, j]$ which is different from QCD convention.
}

$S_{\mathrm{YM}}^{(0)} \equiv \sum_{a=1, n} \frac{E_{\mu} k_{a}^{\mu}}{k_{s} \cdot k_{a}}$

while the sub-leading soft operator is given by

$S_{\mathrm{YM}}^{(1)} \equiv-i \sum_{a=1, n} \frac{E_{\mu} k_{s v} J_{a}^{\mu \nu}}{k_{s} \cdot k_{a}}$

with $E_{\mu}$ the polarization vector of soft gluon.

In the spinor-helicity formulism, employing the holomorphic soft limit (2.9), the soft gluon theorem (2.14) may be expressed as

$\mathcal{A}_{n+1}\left(\ldots,\left\{\epsilon \lambda_{s}, \tilde{\lambda}_{s}\right\}\right)=\left(\frac{1}{\epsilon^{2}} S^{(0)}+\frac{1}{\epsilon} S^{(1)}\right) \mathcal{A}_{n}+\mathcal{O}\left(\epsilon^{0}\right)$.

Taking the helicity of the soft gluon $h_{s}=+1$ as a convention, the soft operators may be written as

$S_{\mathrm{YM}}^{(0)}=\frac{\langle x, n\rangle}{\langle s, n\rangle\langle x, s\rangle}+\frac{\langle x, 1\rangle}{\langle s, 1\rangle\langle x, s\rangle}$,

$S_{\mathrm{YM}}^{(1)}=\frac{1}{\langle n, s\rangle} \tilde{\lambda}_{s \dot{\alpha}} \frac{\partial}{\partial \tilde{\lambda}_{n \dot{\alpha}}}+\frac{1}{\langle s, 1\rangle} \tilde{\lambda}_{s \dot{\alpha}} \frac{\partial}{\partial \tilde{\lambda}_{1 \dot{\alpha}}}$,

with $\lambda_{x}, \lambda_{y}$ arbitrary choosen reference spinors and the freedom in this choice is equivalent to the gauge freedom.

It is important to note that two amplitudes in the soft theorem are both unstripped. In other words, amplitudes $\mathcal{A}_{n+1}$ and $\mathcal{A}_{n}$ in Eq. (2.17) contain respective momentum conservation delta functions. With this in mind, we can remove dependence of anti-holomorphic spinors $\tilde{\lambda}_{1}$ and $\tilde{\lambda}_{n}$ in these two amplitudes by imposing momentum conservation delta functions appropriately. This implies that the sub-leading soft divergence vanishes in color-ordered Yang-Mills amplitudes [4].

As we will see immediately, however, that the operator $S_{\text {YM }}^{(1)}$ is necessary for constructing gravity soft operators from Yang-Mills soft operators.

\subsection{Gravity soft operators as double copy of Yang-Mills soft operators}

There exists a remarkable relation between gravity amplitudes and Yang-Mills amplitudes. At tree level, Kawai, Lewellen and Tye (KLT) found that one can express a closed string amplitude as a sum of the square of open string amplitudes [72]. In field theory limit, this relation expresses a gravity amplitude as a sum of the square of color-ordered YangMills amplitudes. The similar relation also exists between gravity soft operators and Yang-Mills soft operators. In [3], the gravity soft operators were expressed as a double copy of Yang-Mills soft operators with a special gauge choice which 
associated with the special choice of shifted external legs in $\mathrm{BCFW}$ recursion. In this subsection, we rewrite this relation with gauge freedom.

First of all, for the sake of convenience, introduce two notations $^{2}$

$\mathfrak{S}^{0}(x, s, a) \equiv \frac{E_{\mu}^{+}\left(\lambda_{x}\right) k_{a}^{\mu}}{k_{s} \cdot k_{a}}=\frac{\langle x, a\rangle}{\langle x, s\rangle\langle s, a\rangle}$,

$\mathfrak{S}^{1}(s, a) \equiv-i \frac{E_{\mu}^{+}\left(\lambda_{x}\right) k_{s v} J_{a}^{\mu \nu}}{k_{s} \cdot k_{a}}=\frac{1}{\langle s, a\rangle} \tilde{\lambda}_{s \dot{\alpha}} \frac{\partial}{\partial \tilde{\lambda}_{a \dot{\alpha}}}$,

which are the fundamental building blocks for constructing gravity soft operators. Employing these notations, one can write the Yang-Mills soft operators as

$S_{\mathrm{YM}}^{(0)}=\mathfrak{S}^{0}(x, s, n)+\mathfrak{S}^{0}(x, s, 1)$,

$S_{\mathrm{YM}}^{(1)}(1, s, n)=\mathfrak{S}^{1}(s, 1)-\mathfrak{S}^{1}(s, n)$.

Let us note a simple relation that expresses a graviton polarization tensor as the product of gluon polarization vectors with same momentum, i.e.,

$E_{\mu \nu}^{ \pm}(k)=E_{\mu}^{ \pm}(k) \times E_{v}^{ \pm}(k)+E_{v}^{ \pm}(k) \times E_{\mu}^{ \pm}(k)$.

Here $E_{\mu \nu}$ have been written in a symmetric form. By making use of this relation, the leading soft operator in gravity can be written as:

$$
\begin{aligned}
S^{(0)} & =\sum_{a=1}^{n} \frac{2\left(E_{\mu}^{+}\left(\lambda_{x}\right) E_{v}^{+}\left(\lambda_{y}\right)\right) k_{a}^{\mu} k_{a}^{v}}{k_{s} \cdot k_{a}} \\
& =\sum_{a=1}^{n}\left(2 k_{s} \cdot k_{a}\right)\left(\frac{E_{\mu}^{+}\left(\lambda_{x}\right) k_{a}^{\mu}}{k_{s} \cdot k_{a}}\right)\left(\frac{E_{v}^{+}\left(\lambda_{y}\right) k_{a}^{v}}{k_{s} \cdot k_{a}}\right) \\
& =\sum_{a=1}^{n} s_{s a} \mathfrak{S}^{0}(x, s, a) \mathfrak{S}^{0}(y, s, a)
\end{aligned}
$$

where $s_{s a}=2 k_{s} \cdot k_{a}=\langle s, a\rangle[s, a]$ and the $\lambda_{x}$ and $\lambda_{y}$ are arbitrary reference spinors and the freedom in this choice is equivalent to the gauge freedom. This relation was presented in [73-75] and derived in $[19,76]$ by KLT realtion $[72,77,78]$.

The sub-leading soft graviton operator can be expressed as

$$
\begin{aligned}
S^{(1)} & =-i \sum_{a=1}^{n}\left(k_{s} \cdot k_{a}\right)\left(\frac{E_{\mu}^{+}\left(\lambda_{x}\right) k_{a}^{\mu}}{k_{s} \cdot k_{a}} \frac{E_{\nu}^{+}\left(\lambda_{y}\right) k_{s \sigma} J_{a}^{\sigma \nu}}{k_{s} \cdot k_{a}}+(x \leftrightarrow y)\right) \\
& =\frac{1}{2} \sum_{a=1}^{n} s_{s a}\left(\mathfrak{S}^{0}(x, s, a)+\mathfrak{S}^{0}(y, s, a)\right) \mathfrak{S}^{1}(s, a)
\end{aligned}
$$

This relation with a special gauge choice was derived in [19] by KLT realtion $[72,77,78]$.

\footnotetext{
${ }^{2}$ Here the first notation $\mathfrak{S}^{0}$ is just famous eikonal factor in Yang-Mills amplitudes $[39,73,74]$ and the ' $x$ ' denotes the reference spinor $\lambda_{x}$ in spinor representation $E_{\alpha \dot{\alpha}}^{+}\left(\lambda_{s}, \tilde{\lambda}_{s}, \lambda_{x}\right)$ of the polarization vector $E_{\mu}^{+}$ [40].
}

Similarly, the sub-sub-leading soft operator may be written as

$S^{(2)}=\frac{1}{2} \sum_{a=1}^{n} s_{s a} \mathfrak{S}^{1}(s, a) \mathfrak{S}^{1}(s, a)$

It is important to notice that here the operator product $\mathfrak{S}^{1}(s, a) \mathfrak{S}^{1}$

$(s, a)$ should be understood as:

$\mathfrak{S}^{1}(s, a) \mathfrak{S}^{1}(s, a)=\frac{1}{\langle a, s\rangle^{2}} \tilde{\lambda}_{s \dot{\alpha}} \tilde{\lambda}_{s \dot{\beta}} \frac{\partial^{2}}{\partial \tilde{\lambda}_{a \dot{\alpha}} \partial \tilde{\lambda}_{a \dot{\beta}}}$.

In another words, the differential only acts on the amplitudes.

\section{Soft theorem in $\mathcal{N}=4$ super-Yang-Mills theory}

We turn to study the soft theorems in supersymmetric theories. In this section, we present the soft theorem in $\mathcal{N}=4$ super-Yang-Mills theory with a rigorous proof at tree level. We also give some lower-point amplitudes examples to demonstrate the validity of super soft theorem, in particular the soft gluino divergence.

Let us begin with a very brief introduction of $\mathcal{N}=4$ SYM and the superamplitudes in the on-shell superspace. The $\mathcal{N}=4$ on-shell field consists of 8 bosons and 8 fermions and one can write it out as

$$
\begin{array}{cl}
h=1: & 1 \text { gluon } g^{+} \\
h=\frac{1}{2}: & 4 \text { gluinos } \Gamma_{A} \\
h=0: & 6 \text { scalars } S_{A B} \\
h=-\frac{1}{2}: & 4 \text { gluinos } \bar{\Gamma}^{A} \\
h=-1: & 1 \text { gluon } g^{-}
\end{array}
$$

Here $A, B, \ldots=1,2,3,4$ are $\mathrm{SU}(4) \mathrm{R}$-symmetry indices and the scalar $S_{A B}$ is antisymmetric in indices $A, B$. The $\mathcal{N}=4$ on-shell superfield can be expanded as follows [79]:

$$
\begin{aligned}
\Phi(p, \eta)= & g^{+}(p)+\eta^{A} \Gamma_{A}(p)+\frac{1}{2 !} \eta^{A} \eta^{B} S_{A B}(p) \\
& +\frac{1}{3 !} \eta^{A} \eta^{B} \eta^{C} \epsilon_{A B C D} \bar{\Gamma}^{D}(p)+\eta^{1} \eta^{2} \eta^{3} \eta^{4} g^{-}(p) .
\end{aligned}
$$

Here Grassmann odd variables $\eta^{A}$ transforms in a fundamental representation of the $\mathrm{SU}(4) \mathrm{R}$-symmetry.

In super-momentum space, a color-ordered superamplitude is a function of spinors $\lambda_{a}, \tilde{\lambda}_{a}$ (or momentum $p_{a}$ ) and Grassmann variables $\eta_{a}$, i.e.,

$$
\begin{aligned}
\mathscr{A}_{n} & \equiv \mathscr{A}_{n}\left(\Phi_{1}, \ldots, \Phi_{n}\right) \\
& =\mathscr{A}_{n}\left(\left\{\lambda_{1}, \tilde{\lambda}_{1}, \eta_{1}\right\}, \ldots,\left\{\lambda_{n}, \tilde{\lambda}_{n}, \eta_{n}\right\}\right) .
\end{aligned}
$$


The component field amplitudes are then obtained by projecting upon the relevant terms in the $\eta_{i}$ expansion of the superamplitude. For detail, see [54-56].

\subsection{Super soft theorem in $\mathcal{N}=4 \mathrm{SYM}$}

Here we derive the soft theorem in $\mathcal{N}=4$ SYM with the help of super-BCFW recursion relation $[34,35,56]$ in the spinorhelicity formulism $[39,41,80]$. Let us choose the soft particle and its adjacent particle to shift:
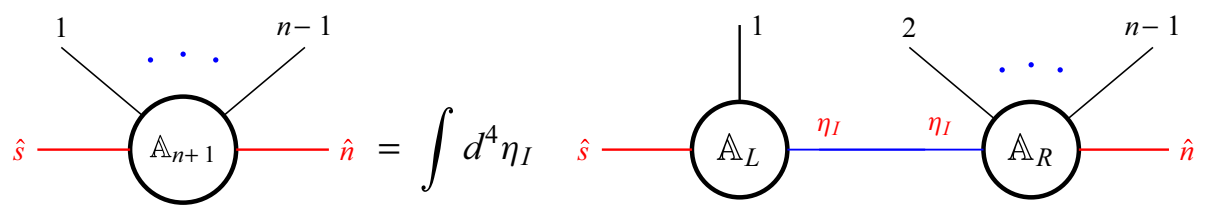
and write

Graphically,

right hand side in Eq. (3.7) under the holomorphic soft limit (2.9). So we drop the terms from contributions with $a>1$,

$$
\begin{aligned}
\mathbb{A}_{n+1}= & \int d^{4} \eta_{I} \mathbb{A}_{3}^{\overline{\mathrm{MHV}}}\left(\left\{\hat{s}\left(z^{*}\right), \eta_{s}\right\},\left\{1, \eta_{1}\right\},\left\{I, \eta_{I}\right\}\right) \\
& \times \frac{1}{P_{I}^{2}} \mathbb{A}_{n}\left(\left\{-I, \eta_{I}\right\},\left\{2, \eta_{2}\right\}, \ldots,\left\{\hat{n}\left(z^{*}\right), \eta_{n}\left(z^{*}\right)\right\}\right) .
\end{aligned}
$$

Here we have

$\lambda_{s}(z)=\lambda_{s}+z \lambda_{n}, \quad \tilde{\lambda}_{n}(z)=\tilde{\lambda}_{n}-z \tilde{\lambda}_{s}, \quad \eta_{n}(z)=\eta_{n}-z \eta_{s}$.

These shifts preserve the total momentum and super-momentum. Super-BCFW recursion gives:

$$
\begin{aligned}
\mathbb{A}_{n+1}= & \sum_{a=1}^{n-2} \int d^{4} \eta_{I} \mathbb{A}_{L}\left(\left\{\lambda_{s}\left(z^{*}\right), \tilde{\lambda}_{s}, \eta_{s}\right\}, 1, \ldots, a,\left\{I\left(z^{*}\right), \eta_{I}\right\}\right) \\
& \times \frac{1}{P_{I}^{2}} \mathbb{A}_{R}\left(\left\{-I\left(z^{*}\right), \eta_{I}\right\}, a+1, \ldots, n-1\right. \\
& \left.\left\{\lambda_{n}, \tilde{\lambda}_{n}\left(z^{*}\right), \eta_{n}\left(z^{*}\right)\right\}\right) .
\end{aligned}
$$

Here the integral over $\eta_{I}$ denotes the sum over intermediate states in ordinary BCFW recursion [56]. The blackboardbold style denotes the stripped superamplitude,

$\mathscr{A}_{n}=\mathbb{A}_{n} \delta^{4}(p)$.

According to different Grassmann odd degrees, one can decompose the superamplitude into various $\mathrm{N}^{k} \mathrm{MHV}$ sectors, i.e.,

$$
\begin{aligned}
\mathbb{A}_{n+1}^{\mathrm{N}^{k} \mathrm{MHV}}= & \int d^{4} \eta_{P} \mathbb{A}_{3}^{\overline{\mathrm{MHV}}}\left(z^{*}\right) \frac{1}{P^{2}} \mathbb{A}_{n}^{\mathrm{N}^{k} \mathrm{MHV}}\left(z^{*}\right) \\
& +\sum_{m=0}^{k-1} \sum_{a=4}^{n} \int d^{4} \eta_{P_{a}} \mathbb{A}_{a}^{\mathrm{N}^{m} \mathrm{MHV}}\left(z_{a}\right) \\
& \times \frac{1}{P_{a}^{2}} \mathbb{A}_{n-a+3}^{\mathrm{N}^{(k-m-1)} \mathrm{MHV}}\left(z_{a}\right) .
\end{aligned}
$$

As shown explicitly in [32], the singular terms only come from the term with $a=1$ in Eq. (3.5), or the first term of the

$$
\begin{aligned}
P_{I}^{2} & =\left(k_{s}+k_{1}\right)^{2}=\langle s, 1\rangle[s, 1], \\
z^{*} & =-\frac{\langle s, 1\rangle}{\langle n, 1\rangle}, \\
\lambda_{I} & =\lambda_{1}, \\
\tilde{\lambda}_{I} & =\tilde{\lambda}_{1}+\frac{\langle n, s\rangle}{\langle n, 1\rangle} \tilde{\lambda}_{s} .
\end{aligned}
$$

In on-shell resursions of tree-level amplitudes, three-point amplitudes are seeds for generating higher-point amplitudes. In on-shell superspace, the three-point superamplitudes of $\mathcal{N}=4 \mathrm{SYM}$ are given by

$\mathscr{A}_{3}^{\mathrm{MHV}}=\frac{\delta^{4}(p)}{\langle 12\rangle\langle 23\rangle\langle 31\rangle} \delta^{8}\left(\sum_{a=1}^{3} \lambda_{a}^{\alpha} \eta_{a}^{A}\right)$
$\mathscr{A}_{3}^{\overline{\mathrm{MHV}}}=\frac{\delta^{4}(p)}{[12][23][31]} \delta^{4}\left([12] \eta_{3}^{A}+[23] \eta_{1}^{A}+[31] \eta_{2}^{A}\right)$.

Then it is easy to get the left three-point superamplitude in Eq. (3.8)

$$
\begin{aligned}
& \mathbb{A}_{3}^{\overline{\mathrm{MHV}}}\left(\left\{\lambda_{s}\left(z^{*}\right), \tilde{\lambda}_{s}, \eta_{s}\right\},\left\{1, \eta_{1}\right\},\left\{I, \eta_{I}\right\}\right) \\
& =\frac{\langle n, 1\rangle[s, 1]}{\langle n, s\rangle} \delta^{4}\left(\eta_{I}-\frac{\langle n, s\rangle}{\langle n, 1\rangle} \eta_{s}-\eta_{1}\right) .
\end{aligned}
$$

Notice that the left superamplitude in Eq. (3.8) is 3-point anti-MHV one. In fact, this corresponds to the super-shift (3.4) and in this case the helicity of soft gluon takes positive one [63]. Inserting the 3-point superamplitude (3.15) into Eq. (3.8) and computing the integral over $\eta_{I}$ give 


$$
\begin{aligned}
\mathbb{A}_{n+1}= & \frac{\langle n, 1\rangle}{\langle n, s\rangle\langle s, 1\rangle} \mathbb{A}_{n}\left(\left\{\lambda_{1}, \tilde{\lambda}_{1}+\frac{\langle n, s\rangle}{\langle n, 1\rangle} \tilde{\lambda}_{s},\right.\right. \\
& \left.\eta_{1}+\frac{\langle n, s\rangle}{\langle n, 1\rangle} \eta_{s}\right\},\left\{\lambda_{2}, \tilde{\lambda}_{2}, \eta_{2}\right\}, \ldots, \\
& \left.\left\{\lambda_{n}, \tilde{\lambda}_{n}+\frac{\langle s, 1\rangle}{\langle n, 1\rangle} \tilde{\lambda}_{s}, \eta_{n}+\frac{\langle s, 1\rangle}{\langle n, 1\rangle} \eta_{s}\right\}\right) .
\end{aligned}
$$

Dressing both sides of the above equation in respective appropriate momentum conservation delta functions, one obtains

$$
\begin{aligned}
\mathscr{A}_{n+1}= & \frac{\langle n, 1\rangle}{\langle n, s\rangle\langle s, 1\rangle} \mathscr{A}_{n}\left(\left\{\lambda_{1}, \tilde{\lambda}_{1}+\frac{\langle n, s\rangle}{\langle n, 1\rangle} \tilde{\lambda}_{s}, \eta_{1}+\frac{\langle n, s\rangle}{\langle n, 1\rangle} \eta_{s}\right\}\right. \\
& \left\{\lambda_{2}, \tilde{\lambda}_{2}, \eta_{2}\right\}, \ldots,\left\{\lambda_{n}, \tilde{\lambda}_{n}+\frac{\langle s, 1\rangle}{\langle n, 1\rangle} \tilde{\lambda}_{s}, \eta_{n}\right. \\
& \left.\left.+\frac{\langle s, 1\rangle}{\langle n, 1\rangle} \eta_{s}\right\}\right) .
\end{aligned}
$$

In the holomorphic soft limit (2.9),

$$
\begin{aligned}
\mathscr{A}_{n+1}(\epsilon)= & \frac{1}{\epsilon^{2}} \frac{\langle n, 1\rangle}{\langle n, s\rangle\langle s, 1\rangle} \mathscr{A}_{n}\left(\left\{\lambda_{1}, \tilde{\lambda}_{1}+\epsilon \frac{\langle n, s\rangle}{\langle n, 1\rangle} \tilde{\lambda}_{s}, \eta_{1}\right.\right. \\
& \left.+\epsilon \frac{\langle n, s\rangle}{\langle n, 1\rangle} \eta_{s}\right\}, \\
& \left\{\lambda_{2}, \tilde{\lambda}_{2}, \eta_{2}\right\}, \ldots,\left\{\lambda_{n}, \tilde{\lambda}_{n}+\epsilon \frac{\langle s, 1\rangle}{\langle n, 1\rangle} \tilde{\lambda}_{s}, \eta_{n}\right. \\
& \left.\left.+\epsilon \frac{\langle s, 1\rangle}{\langle n, 1\rangle} \eta_{s}\right\}\right) .
\end{aligned}
$$

Performing Taylor expansion at $\epsilon=0$, we obtain the soft theorem

$\mathscr{A}_{n+1}(\epsilon)=\left(\frac{1}{\epsilon^{2}} \mathcal{S}_{\mathrm{SYM}}^{(0)}+\frac{1}{\epsilon} \mathcal{S}_{\mathrm{SYM}}^{(1)}\right) \mathscr{A}_{n}+\mathcal{O}\left(\epsilon^{0}\right)$

where

$$
\begin{aligned}
\mathcal{S}_{\mathrm{SYM}}^{(0)} & =\frac{\langle n, 1\rangle}{\langle n, s\rangle\langle s, 1\rangle}=S_{\mathrm{YM}}^{(0)}, \\
\mathcal{S}_{\mathrm{SYM}}^{(1)} & =S_{\mathrm{YM}}^{(1)}+\eta_{s}^{A} \mathcal{F}_{A}^{(0)}, \\
\mathcal{F}_{A}^{(0)} & \equiv \frac{1}{\langle s, 1\rangle} \frac{\partial}{\partial \eta_{1}^{A}}+\frac{1}{\langle n, s\rangle} \frac{\partial}{\partial \eta_{n}^{A}} .
\end{aligned}
$$

Let us expand the superamplitude $\mathscr{A}_{n+1}$ in Grassmannian variables $\eta_{s}$

$$
\begin{aligned}
\mathscr{A}_{n+1}\left(\Phi_{1}, \ldots, \Phi_{n}, \Phi_{s}\right) \\
=\mathscr{A}_{n+1}\left(\Phi_{1}, \ldots, \Phi_{n}, g_{s}^{+}\right)+\eta_{s}^{A} \mathscr{A}_{n+1}\left(\Phi_{1}, \ldots, \Phi_{n}, \Gamma_{s A}\right) \\
\quad+\frac{1}{2 !} \eta_{s}^{A} \eta_{s}^{B} \mathscr{A}_{n+1}\left(\Phi_{1}, \ldots, \Phi_{n}, S_{s A B}\right)+\cdots
\end{aligned}
$$

According to the degrees of the Grassmann odd $\eta_{s}$, we can express super soft theorem (3.19) as following:

$$
\begin{aligned}
\mathscr{A}_{n+1}\left(\ldots, g_{s}^{+}\right)(\epsilon) & =\left(\frac{1}{\epsilon^{2}} S_{\mathrm{YM}}^{(0)}+\frac{1}{\epsilon} S_{\mathrm{YM}}^{(1)}\right) \mathscr{A}_{n}+\mathcal{O}\left(\epsilon^{0}\right), \\
\mathscr{A}_{n+1}\left(\ldots, \Gamma_{s A}\right)(\epsilon) & =\frac{1}{\epsilon} \mathcal{F}_{A}^{(0)} \mathscr{A}_{n}+\mathcal{O}\left(\epsilon^{0}\right), \\
\mathscr{A}_{n+1}\left(\ldots, S_{S A B}\right)(\epsilon) & =\frac{0}{\epsilon}+\mathcal{O}\left(\epsilon^{0}\right) .
\end{aligned}
$$

In the last equation, the term $\frac{0}{\epsilon}$ implies that there is no singular term. The soft gluon operators in $\mathcal{N}=4 \mathrm{SYM}$ are identical to the ones in pure Yang-Mills. As mentioned in Sect. 2, the sub-leading soft gluon divergence is also vanishing in $\mathcal{N}=4 \mathrm{SYM}$. As we expected, the amplitudes involve more types of particle, including gluon, gluino and scalar in $\mathcal{N}=4$ SYM. More interestingly, we find the soft divergence of amplitudes involving a soft fermionic gluino. Notice that the leading soft gluino operator $\mathcal{F}_{A}^{(0)}$ involves the first order derivative with respect to the Grassmannian variables $\eta_{1}$ and $\eta_{n}$. In fact, these two terms of $\mathcal{F}_{A}^{(0)}$ change helicity of corresponding external leg in respectively. And this preserves the total helicity as well as SU(4) R-symmetry before and after soft gluino emission. We also provide an alternative derivation of the soft theorem in $\mathcal{N}=4 \mathrm{SYM}$ in Appendix A. In the next subsection, we will check soft theorem by some examples in detail. We will pay special attention to soft gluino theorem.

\subsection{MHV examples}

In the remainder of this section, we verify the soft theorem presented above by some examples in detail. We take special care of the property of amplitudes when a gluino leg becomes soft.

The simplest example is MHV sector. In this sector, one can study the amplitudes involving an arbitrary number of external legs. In the holomorphic soft limit $\lambda_{s} \rightarrow \epsilon \lambda_{s}$, the soft theorem of pure gluonic MHV amplitudes gives

$\mathcal{A}_{n+1}^{\mathrm{MHV}}(\epsilon)=\frac{1}{\epsilon^{2}} S_{\mathrm{YM}}^{(0)} \mathcal{A}_{n}^{\mathrm{MHV}}$

which is exact in $\epsilon$.

Now we study the amplitude $\mathcal{A}\left(\bar{\Gamma}^{A}, g^{+}, \ldots, g^{+}, g^{-}, \Gamma_{B}\right)$ involving a gluino-anti-gluino pair. Using the soft gluino theorem (3.24), we get

$$
\begin{aligned}
& \mathcal{A}_{n+1}\left(\bar{\Gamma}^{A}, g^{+}, \ldots, g^{+}, g^{-}, \Gamma_{B}\right) \\
& \quad=\delta_{B}^{A} \frac{1}{\epsilon} \frac{1}{\langle s, 1\rangle} \mathcal{A}_{n}\left(g^{-}, g^{+}, \ldots, g^{+}, g^{-}\right)+\mathcal{O}\left(\epsilon^{0}\right) .
\end{aligned}
$$

in the holomorphic soft limit $\lambda_{s} \rightarrow \epsilon \lambda_{s}$. We will show that there is no $\mathcal{O}\left(\epsilon^{0}\right)$ corrections in above relation. Noticing the supersymmetry Ward identity (SWI) $[39,54]$ : 


$$
\begin{aligned}
& \mathcal{A}_{n+1}\left(\bar{\Gamma}^{A}, g^{+}, \ldots, g^{+}, g_{n}^{-}, \Gamma_{B}\right) \\
& \quad=\delta_{B}^{A} \frac{\langle n, s\rangle}{\langle n, 1\rangle} \mathcal{A}_{n+1}\left(g^{-}, g^{+}, \ldots, g^{+}, g^{-}, g^{+}\right)
\end{aligned}
$$

then using the soft gluon theorem (3.26), we have

$$
\begin{aligned}
\delta_{B}^{A} & \frac{\epsilon\langle n, s\rangle}{\langle n, 1\rangle} \mathcal{A}_{n+1}\left(g^{-}, g^{+}, \ldots, g^{+}, g^{-}, g^{+}\right) \\
& =\delta_{B}^{A} \frac{\epsilon\langle n, s\rangle}{\langle n, 1\rangle} \times \frac{1}{\epsilon^{2}} \frac{\langle n, 1\rangle}{\langle n, s\rangle\langle s, 1\rangle} \mathcal{A}_{n}\left(g^{-}, g^{+}, \ldots, g^{+}, g^{-}\right) \\
& =\delta_{B}^{A} \frac{1}{\epsilon} \frac{1}{\langle s, 1\rangle} \mathcal{A}_{n}\left(g^{-}, g^{+}, \ldots, g^{+}, g^{-}\right)
\end{aligned}
$$

in the holomorphic soft limit $\lambda_{s} \rightarrow \epsilon \lambda_{s}$. This agrees with Eq. (3.27).

In the MHV sector, another a SWI involving two scalars is $[39,54]$ :

$$
\begin{aligned}
& \mathcal{A}_{n+1}\left(S_{12}, g^{-}, g^{+}, \ldots, g^{+}, S_{34}\right) \\
& \quad=\frac{\langle 2, s\rangle^{2}}{\langle 2,1\rangle^{2}} \mathcal{A}_{n+1}\left(g^{-}, g^{-}, g^{+}, \ldots, g^{+}, g^{+}\right) .
\end{aligned}
$$

Using the soft gluon theorem (3.26) for the right hand side of above equation, one finds that

$$
\begin{aligned}
& \mathcal{A}_{n+1}\left(S_{12}, g^{-}, g^{+}, \ldots, g^{+}, S_{34}\right) \\
& \quad \stackrel{\lambda_{s} \rightarrow \epsilon \lambda_{s}}{=} \frac{\langle 2, s\rangle^{2}}{\langle 2,1\rangle^{2}} S_{\mathrm{YM}}^{(0)} \mathcal{A}_{n}\left(g^{-}, g^{-}, g^{+}, \ldots, g^{+}\right) \\
& =\mathcal{A}_{n+1}\left(S_{12}, g^{-}, g^{+}, \ldots, g^{+}, S_{34}\right) \sim \mathcal{O}\left(\epsilon^{0}\right) .
\end{aligned}
$$

It agrees with the soft theorem (3.25). This also shows that the MHV amplitudes involving two scalars remain invariant under rescaling of momentum of one of scalars.

\subsection{Six-point NMHV examples}

Next we turn to the Next-to-MHV (NMHV) sector. In this sector, it is difficult to check the amplitudes which consist of an arbitrary number of external legs. Here we mainly check 6point NMHV amplitudes involving a gluino-anti-gluino pair which were obtained by Feynman diagrams [81], also by solving supersymmetry Ward identity [82] and BCFW recursion [83].

The first example is $A_{6}\left(g_{1}^{-}, g_{2}^{-}, \bar{\Gamma}_{3}^{A}, \Gamma_{4 B}, g_{5}^{+}, g_{6}^{+}\right)$:

$$
\begin{aligned}
A_{6}\left(g_{1}^{-}, g_{2}^{-}, \bar{\Gamma}_{3}^{A}, \Gamma_{4 B}, g_{5}^{+}, g_{6}^{+}\right) \\
=-\frac{[4|2+3| 1\rangle^{2}[3|2+4| 1\rangle}{s_{234}[23][34]\langle 56\rangle\langle 61\rangle[2|3+4| 5\rangle} \delta_{B}^{A} \\
\quad+\frac{[6|1+2| 3\rangle^{2}[6|1+2| 4\rangle}{s_{612}[61][12]\langle 34\rangle\langle 45\rangle[2|6+1| 5\rangle} \delta_{B}^{A} .
\end{aligned}
$$

In the soft limit $\lambda_{4} \rightarrow \epsilon \lambda_{4}$,

$$
\begin{aligned}
A_{6} & \left(g_{1}^{-}, g_{2}^{-}, \bar{\Gamma}_{3}^{A}, \Gamma_{4 B}, g_{5}^{+}, g_{6}^{+}\right) \\
= & \delta_{B}^{A} \frac{1}{\epsilon} \frac{[65]^{2}\langle 53\rangle^{2}[6|3+5| 4\rangle}{s_{35}[61][12]\langle 34\rangle\langle 45\rangle[23]\langle 35\rangle}+\mathcal{O}\left(\epsilon^{0}\right) \\
= & \delta_{B}^{A} \frac{1}{\epsilon}\left(\frac{1}{\langle 34\rangle} A_{5}\left(g_{1}^{-}, g_{2}^{-}, g_{3}^{-}, g_{5}^{+}, g_{6}^{+}\right)\right. \\
& \left.+\frac{1}{\langle 45\rangle} A_{5}\left(g_{1}^{-}, g_{2}^{-}, \bar{\Gamma}_{3}^{C}, \Gamma_{5 C}, g_{6}^{+}\right)\right)+\mathcal{O}\left(\epsilon^{0}\right)
\end{aligned}
$$

This agrees completely with the soft gluino theorem (3.24). Here we have used two 5-point amplitudes follows:

$A_{5}\left(g_{1}^{-}, g_{2}^{-}, g_{3}^{-}, g_{5}^{+}, g_{6}^{+}\right)=\frac{[56]^{4}}{[12][23][35][56][61]}$,
$A_{5}\left(g_{1}^{-}, g_{2}^{-}, \bar{\Gamma}_{3}^{A}, \Gamma_{5 B}, g_{6}^{+}\right)=\frac{[65]^{2}[63]}{[12][23][35][61]} \delta_{B}^{A}$.

The second example is the amplitude:

$$
\begin{aligned}
& A_{6}\left(g_{1}^{-}, \bar{\Gamma}_{2}^{A}, g_{3}^{-}, \Gamma_{4 B}, g_{5}^{+}, g_{6}^{+}\right) \\
& =-\frac{[4|2+3| 1\rangle^{2}[2|3+4| 1\rangle}{s_{234}[23][34]\langle 56\rangle\langle 61\rangle[2|3+4| 5\rangle} \delta_{B}^{A} \\
& \quad+\frac{[6|1+2| 3\rangle^{2}[26]\langle 34\rangle}{s_{612}[61][12]\langle 34\rangle\langle 45\rangle[2|6+1| 5\rangle} \delta_{B}^{A} .
\end{aligned}
$$

In the soft limit $\lambda_{4} \rightarrow \epsilon \lambda_{4}$, we have

$$
\begin{aligned}
& A_{6}\left(g_{1}^{-}, \bar{\Gamma}_{2}^{A}, g_{3}^{-}, \Gamma_{4 B}, g_{5}^{+}, g_{6}^{+}\right) \\
& \quad=\delta_{B}^{A} \frac{1}{\epsilon} \frac{[65]^{2}\langle 53\rangle^{2}[26]}{s_{35}[61][12]\langle 45\rangle[23]\langle 35\rangle}+\mathcal{O}\left(\epsilon^{0}\right) \\
& \quad=\delta_{B}^{A} \frac{1}{\epsilon} \frac{1}{\langle 45\rangle} A_{5}\left(g_{1}^{-}, \bar{\Gamma}_{2}^{A}, g_{3}^{-}, \Gamma_{5 A}, g_{6}^{+}\right)+\mathcal{O}\left(\epsilon^{0}\right)
\end{aligned}
$$

where

$$
A_{5}\left(g_{1}^{-}, \bar{\Gamma}_{2}^{A}, g_{3}^{-}, \Gamma_{5 B}, g_{6}^{+}\right)=\frac{[56]}{[26]} \frac{[56]^{4}}{[12][23][35][56][61]} \delta_{B}^{A} .
$$

This also agrees with the soft gluino theorem (3.24). Similarly, after some calculation we have

$$
\begin{aligned}
& A_{6}\left(\bar{\Gamma}_{1}^{A}, g_{2}^{-}, g_{3}^{-}, \Gamma_{4 B}, g_{5}^{+}, g_{6}^{+}\right) \\
& \quad \stackrel{\lambda_{4} \rightarrow \epsilon \lambda_{4}}{=} \frac{1}{\epsilon} \frac{1}{\langle 45\rangle} A_{5}\left(\bar{\Gamma}_{1}^{A}, g_{2}^{-}, g_{3}^{-}, \Gamma_{5 B}, g_{6}^{+}\right)+\mathcal{O}\left(\epsilon^{0}\right), \\
& A_{6}\left(g_{1}^{-}, \bar{\Gamma}_{2}^{A}, g_{3}^{-}, g_{4}^{+}, \Gamma_{5 B}, g_{6}^{+}\right) \\
& \quad \stackrel{\lambda_{5} \rightarrow \epsilon \lambda_{5}}{=} \frac{1}{\epsilon} \frac{1}{\langle 45\rangle} A_{5}\left(g_{1}^{-}, \bar{\Gamma}_{2}^{A}, g_{3}^{-}, \Gamma_{4 B}, g_{6}^{+}\right) \\
& \quad+\frac{1}{\epsilon} \frac{1}{\langle 56\rangle} A_{5}\left(g_{1}^{-}, \bar{\Gamma}_{2}^{A}, g_{3}^{-}, g_{4}^{+}, \Gamma_{6 B}\right)+\mathcal{O}\left(\epsilon^{0}\right),
\end{aligned}
$$




$$
\begin{aligned}
& A_{6}\left(\bar{\Gamma}_{1}^{A}, g_{2}^{-}, g_{3}^{-}, g_{4}^{+}, \Gamma_{5 B}, g_{6}^{+}\right) \\
& \quad \stackrel{\lambda_{5} \rightarrow \epsilon \lambda_{5}}{=} \frac{1}{\epsilon} \frac{1}{\langle 45\rangle} A_{5}\left(\bar{\Gamma}_{1}^{A}, g_{2}^{-}, g_{3}^{-}, \Gamma_{4 B}, g_{6}^{+}\right) \\
& \quad+\frac{1}{\epsilon} \frac{1}{\langle 56\rangle} A_{5}\left(\bar{\Gamma}_{1}^{A}, g_{2}^{-}, g_{3}^{-}, g_{4}^{+}, \Gamma_{6 B}\right)+\mathcal{O}\left(\epsilon^{0}\right),
\end{aligned}
$$

$$
\begin{gathered}
A_{6}\left(\bar{\Gamma}_{1}^{A}, g_{2}^{-}, \Gamma_{3 B}, g_{4}^{-}, g_{5}^{+}, g_{6}^{+}\right) \\
\stackrel{\lambda_{3} \rightarrow \epsilon \lambda_{3}}{=} \frac{0}{\epsilon}+\mathcal{O}\left(\epsilon^{0}\right),
\end{gathered}
$$

$$
\begin{aligned}
& A_{6}\left(g_{1}^{-}, \bar{\Gamma}_{2}^{A}, \Gamma_{3 B}, g_{4}^{-}, g_{5}^{+}, g_{6}^{+}\right) \\
& \stackrel{\lambda_{3} \rightarrow \epsilon \lambda_{3}}{\underline{\epsilon}} \frac{1}{\epsilon} \frac{1}{\langle 23\rangle} \delta_{B}^{A} A_{5}\left(g_{1}^{-}, g_{2}^{-}, g_{4}^{-}, g_{5}^{+}, g_{6}^{+}\right)+\mathcal{O}\left(\epsilon^{0}\right),
\end{aligned}
$$

$$
\begin{aligned}
& A_{6}\left(g_{1}^{-}, g_{2}^{-}, \Gamma_{3 B}, \bar{\Gamma}_{4}^{A}, g_{5}^{+}, g_{6}^{+}\right) \\
& \stackrel{\lambda_{3} \rightarrow \epsilon \lambda_{3}}{=} \frac{1}{\epsilon} \frac{1}{\langle 34\rangle} \delta_{B}^{A} A_{5}\left(g_{1}^{-}, g_{2}^{-}, g_{4}^{-}, g_{5}^{+}, g_{6}^{+}\right)+\mathcal{O}\left(\epsilon^{0}\right),
\end{aligned}
$$

$$
\begin{aligned}
& A_{6}\left(\bar{\Gamma}_{1}^{A}, \Gamma_{2 B}, g_{3}^{-}, g_{4}^{+}, g_{5}^{-}, g_{6}^{+}\right) \\
& \quad \stackrel{\lambda_{2} \rightarrow \epsilon \lambda_{2}}{=} \frac{1}{\epsilon} \frac{1}{\langle 12\rangle} \delta_{B}^{A} A_{5}\left(g_{1}^{-}, g_{3}^{-}, g_{4}^{+}, g_{5}^{-}, g_{6}^{+}\right)+\mathcal{O}\left(\epsilon^{0}\right), \\
& A_{6}\left(g_{1}^{-}, \Gamma_{2 B}, \bar{\Gamma}_{3}^{A}, g_{4}^{+}, g_{5}^{-}, g_{6}^{+}\right) \\
& \quad \stackrel{\lambda_{2} \rightarrow \epsilon \lambda_{2}}{=} \frac{1}{\epsilon} \frac{1}{\langle 23\rangle} \delta_{B}^{A} A_{5}\left(g_{1}^{-}, g_{3}^{-}, g_{4}^{+}, g_{5}^{-}, g_{6}^{+}\right)+\mathcal{O}\left(\epsilon^{0}\right),
\end{aligned}
$$

$$
\begin{gathered}
A_{6}\left(g_{1}^{-}, \Gamma_{2 B}, g_{3}^{-}, g_{4}^{+}, \bar{\Gamma}_{5}^{A}, g_{6}^{+}\right) \\
\stackrel{\lambda_{2} \rightarrow \epsilon \lambda_{2}}{=} \frac{0}{\epsilon}+\mathcal{O}\left(\epsilon^{0}\right) .
\end{gathered}
$$

Here we omit some details. These examples strongly support the soft gluino theorem (3.24). In addition, the same checks can be done for other six-point NMHV amplitudes in $\mathcal{N}=4$ SYM, for example tree-level four-gluino two-gluon amplitudes and six-gluino amplitudes [84].

\section{Super soft theorem in $\mathcal{N}=8$ supergravity}

The $\mathcal{N}=8$ Supergravity is the most symmetric quantum field theory in 4 dimensions. In this section, we study super soft theorem in $\mathcal{N}=8$ SUGRA. First we derive the soft theorem by using super-BCFW recursion relations at tree level. Then we verify soft divergences of scattering amplitudes of $\mathcal{N}=8$ SUGRA, in particular soft gravitino and soft gravi-photon divergences, by some MHV tree-level amplitudes exactly. We also give "KLT-like relations" between soft operators in $\mathcal{N}=8$ SUGRA and ones in $\mathcal{N}=4$ SYM at the end of this section.
The $\mathcal{N}=8$ SUGRA consists of 256 massless on-shell fields which can be characterized as

$$
(\mathcal{N}=8 \text { SUGRA }) \sim(\mathcal{N}=4 \text { SYM }) \otimes(\mathcal{N}=4 \text { SYM }) .
$$

These on-shell fields form a CPT-self-conjugate supermultiplet and may be organized into a single on-shell superfield $\Phi$. With the help of the Grassmann odd variables $\eta^{A}$, one can expand on-shell superfield $\Phi$ follows

$$
\begin{aligned}
\Phi(p, \eta)= & h^{+}(p)+\eta^{A} \psi_{A}(p)+\frac{1}{2 !} \eta^{A} \eta^{B} v_{A B}(p) \\
& +\frac{1}{3 !} \eta^{A} \eta^{B} \eta^{C} \chi_{A B C}(p) \\
& +\frac{1}{4 !} \eta^{A} \eta^{B} \eta^{C} \eta^{D} S_{A B C D}(p)+\cdots \\
& +\eta^{1} \eta^{2} \eta^{3} \eta^{4} \eta^{5} \eta^{6} \eta^{7} \eta^{8} h^{-}(p) .
\end{aligned}
$$

Here $A, B, \ldots=1,2, \ldots, \mathcal{N}$ are $\mathrm{SU}(8)$ R-symmetry indices and each state above is fully antisymmetric in these labels.

In $\mathcal{N}=8$ on-shell superspace, there are also fundamental three-point superamplitudes:

$$
\begin{aligned}
& \mathscr{M}_{3}^{\mathrm{MHV}}=\frac{\delta^{4}(p)}{(\langle 12\rangle\langle 23\rangle\langle 31\rangle)^{2}} \delta^{16}\left(\lambda_{1}^{\alpha} \eta_{1}^{A}+\lambda_{2}^{\alpha} \eta_{2}^{A}+\lambda_{3}^{\alpha} \eta_{3}^{A}\right), \\
& \mathscr{M}_{3}^{\overline{\mathrm{MHV}}}=\frac{\delta^{4}(p)}{([12][23][31])^{2}} \delta^{8}\left([12] \eta_{3}^{A}+[23] \eta_{1}^{A}+[31] \eta_{2}^{A}\right) .
\end{aligned}
$$

Here each is just the square of corresponding three-point superamplitude of $\mathcal{N}=4 \mathrm{SYM}$.

\subsection{Super soft theorem in $\mathcal{N}=8$ SUGRA}

Now we start to derive the soft theorem. Consider an on-shell $(n+1)$-point superamplitudes in $\mathcal{N}=8$ SUGRA with a soft external leg $\Phi_{s}\left(\lambda_{s}, \tilde{\lambda}_{s}, \eta_{s}\right)^{3}$

$$
\mathscr{M}_{n+1} \equiv \mathscr{M}_{n+1}\left(\left\{\lambda_{1}, \tilde{\lambda}_{1}, \eta_{1}\right\}, \ldots,\left\{\lambda_{n}, \tilde{\lambda}_{n}, \eta_{n}\right\},\left\{\lambda_{s}, \tilde{\lambda}_{s}, \eta_{s}\right\}\right) .
$$

Let us choose the following super-shift:

$\lambda_{s}(z)=\lambda_{s}+z \lambda_{n}, \quad \tilde{\lambda}_{n}(z)=\tilde{\lambda}_{n}-z \tilde{\lambda}_{s}, \quad \eta_{n}(z)=\eta_{n}-z \eta_{s}$.

Using the analysis similar to SYM, the super-BCFW recursion gives

\footnotetext{
3 Here soft particle may be any one in $\mathcal{N}=8$ supermultiplet (4.1), including graviton (spin-2), gravitino (spin-3/2), gravi-photon (spin-1), gravi-photino (spin-1/2) and scalar (spin-0).
} 


$$
\begin{aligned}
\mathscr{M}_{n+1}= & \sum_{a=1}^{n-1} \frac{[s, a]\langle n, a\rangle^{2}}{\langle s, a\rangle\langle n, s\rangle^{2}} \\
& \times \mathscr{M}_{n}\left(\ldots,\left\{\lambda_{a}, \tilde{\lambda}_{a}+\frac{\langle n, s\rangle}{\langle n, a\rangle} \tilde{\lambda}_{s}, \eta_{a}+\frac{\langle n, s\rangle}{\langle n, a\rangle} \eta_{s}\right\}, \ldots\right. \\
& \left.\left\{\lambda_{n}, \tilde{\lambda}_{n}+\frac{\langle s, a\rangle}{\langle n, a\rangle} \tilde{\lambda}_{s}, \eta_{n}+\frac{\langle s, a\rangle}{\langle n, a\rangle} \eta_{s}\right\}\right) .
\end{aligned}
$$

Here one has omitted terms which stay finite in the holomorphic soft limits (2.9). Applying the deformation $\lambda_{s} \rightarrow \epsilon \lambda_{s}$ to above formula, one gets

$$
\begin{aligned}
\mathscr{M}_{n+1}(\epsilon)= & \frac{1}{\epsilon^{3}} \sum_{a=1}^{n-1} \frac{[s, a]\langle n, a\rangle^{2}}{\langle s, a\rangle\langle n, s\rangle^{2}} \\
& \times \mathscr{M}_{n}\left(\ldots,\left\{\lambda_{a}, \tilde{\lambda}_{a}+\epsilon \frac{\langle n, s\rangle}{\langle n, a\rangle} \tilde{\lambda}_{s}, \eta_{a}\right.\right. \\
& \left.+\epsilon \frac{\langle n, s\rangle}{\langle n, a\rangle} \eta_{s}\right\}, \ldots,\left\{\lambda_{n}, \tilde{\lambda}_{n}+\epsilon \frac{\langle s, a\rangle}{\langle n, a\rangle} \tilde{\lambda}_{s}, \eta_{n}\right. \\
& \left.\left.+\epsilon \frac{\langle s, a\rangle}{\langle n, a\rangle} \eta_{s}\right\}\right) .
\end{aligned}
$$

Performing Taylor expansion of $\mathcal{M}(\epsilon)$ around $\epsilon=0$, one obtains the super soft theorem:

$$
\mathscr{M}_{n+1}(\epsilon)=\left(\frac{1}{\epsilon^{3}} \mathcal{S}^{(0)}+\frac{1}{\epsilon^{2}} \mathcal{S}^{(1)}+\frac{1}{\epsilon} \mathcal{S}^{(2)}\right) \mathscr{M}_{n}+\mathcal{O}\left(\epsilon^{0}\right) .
$$

Here the leading soft factor is same with the one in nonsupersymmetric gravity theory,

$\mathcal{S}^{(0)}=\sum_{a=1}^{n-1} \frac{[s, a]\langle n, a\rangle^{2}}{\langle s, a\rangle\langle n, s\rangle^{2}}=S^{(0)}$.

The sub-leading soft operator consists of two parts:

$$
\begin{aligned}
\mathcal{S}^{(1)} & =\sum_{a=1}^{n-1} \frac{[s, a]\langle n, a\rangle}{\langle s, a\rangle\langle n, s\rangle}\left(\tilde{\lambda}_{s \dot{\alpha}} \frac{\partial}{\partial \tilde{\lambda}_{a \dot{\alpha}}}+\eta_{s}^{A} \frac{\partial}{\partial \eta_{a}^{A}}\right) \\
& =S^{(1)}+\eta_{s}^{A} \mathcal{S}_{A}^{(1)},
\end{aligned}
$$

while the sub-sub-leading soft operator consists of three parts:

$$
\begin{aligned}
\mathcal{S}^{(2)} & =S^{(2)}+\eta_{s}^{A} \mathcal{S}_{A}^{(2)}+\frac{1}{2} \eta_{s}^{A} \eta_{s}^{B} \mathcal{S}_{A B}^{(2)}, \\
S^{(2)} & =\frac{1}{2} \sum_{a=1}^{n} \frac{[s, a]}{\langle s, a\rangle} \tilde{\lambda}_{s \dot{\alpha}} \tilde{\lambda}_{s \dot{\beta}} \frac{\partial^{2}}{\partial \tilde{\lambda}_{a \dot{\alpha}} \partial \tilde{\lambda}_{a \dot{\beta}}}, \\
\mathcal{S}_{A}^{(2)} & =\sum_{a=1}^{n} \frac{[s, a]}{\langle s, a\rangle} \tilde{\lambda}_{s \dot{\alpha}} \frac{\partial^{2}}{\partial \tilde{\lambda}_{a \dot{\alpha}} \partial \eta_{a}^{A}}, \\
\mathcal{S}_{A B}^{(2)} & =\sum_{a=1}^{n} \frac{[s, a]}{\langle s, a\rangle} \frac{\partial^{2}}{\partial \eta_{a}^{B} \partial \eta_{a}^{A}} .
\end{aligned}
$$

See Appendix B for some calculational details. Expanding superamplitude $\mathscr{M}_{n+1}$ in Grassman odd variables $\eta_{s}$, we have

$$
\begin{aligned}
& \mathscr{M}_{n+1}\left(\Phi_{1}, \ldots, \Phi_{n}, \Phi_{s}\right) \\
= & \mathscr{M}_{n+1}\left(\Phi_{1}, \ldots, \Phi_{n}, h_{s}^{+}\right)+\eta_{s}^{A} \mathscr{M}_{n+1}\left(\Phi_{1}, \ldots, \Phi_{n}, \psi_{s A}\right) \\
& +\frac{1}{2} \eta_{s}^{A} \eta_{s}^{B} \mathscr{M}_{n+1}\left(\Phi_{1}, \ldots, \Phi_{n}, v_{s A B}\right)+\cdots .
\end{aligned}
$$

Thus we can express the soft theorem (4.9) in $\mathcal{N}=8$ SUGRA as

soft graviton: $\mathscr{M}_{n+1}\left(\ldots, h_{s}^{+}\right)(\epsilon)$,

$$
=\left(\frac{1}{\epsilon^{3}} S^{(0)}+\frac{1}{\epsilon^{2}} S^{(1)}+\frac{1}{\epsilon} S^{(2)}\right) \mathscr{M}_{n}+\mathcal{O}\left(\epsilon^{0}\right),
$$

soft gravitino: $\mathscr{M}_{n+1}\left(\ldots, \psi_{s A}\right)(\epsilon)$

$$
=\left(\frac{1}{\epsilon^{2}} \mathcal{S}_{A}^{(1)}+\frac{1}{\epsilon} \mathcal{S}_{A}^{(2)}\right) \mathscr{M}_{n}+\mathcal{O}\left(\epsilon^{0}\right),
$$

soft gravi-photon: $\mathscr{M}_{n+1}\left(\ldots, v_{s A B}\right)(\epsilon)$

$$
=\frac{1}{\epsilon} \mathcal{S}_{A B}^{(2)} \mathscr{M}_{n}+\mathcal{O}\left(\epsilon^{0}\right),
$$

soft gravi-photino: $\mathscr{M}_{n+1}\left(\ldots, \chi_{s A B C}\right)(\epsilon)=\frac{0}{\epsilon}+\mathcal{O}\left(\epsilon^{0}\right)$,

soft scalar: $\mathscr{M}_{n+1}\left(\ldots, S_{S A B C D}\right)(\epsilon)=\frac{0}{\epsilon}+\mathcal{O}\left(\epsilon^{0}\right)$.

There are more contents in $\mathcal{N}=8$ SUGRA. Scattering amplitudes of $\mathcal{N}=8$ SUGRA involve more types of particle. That is, every (hard or soft) external leg in amplitudes may be any particle of $4 \mathrm{D} \mathcal{N}=8$ SUGRA. Thus the soft graviton theorem (4.17) in SUGRA incorporates the soft graviton theorem for pure graviton amplitudes. Besides the soft graviton theorem, one obtains leading and sub-leading soft gravitino divergences and leading soft gravi-photon divergence. Also one finds that there are no soft gravi-photino divergence and soft scalar divergence.

In the next subsection, we will check soft theorems by some examples in the MHV sector of $\mathcal{N}=8$ SUGRA in detail. We will pay special attention to the soft gravitino divergence and the soft gravi-photon divergence.

\subsection{MHV examples}

For the soft graviton theorem, in particular leading and subleading orders, there are a great deal of study and investigation on both theoretical derivations and special examples check so far $[20,28,32]$. In this subsection, we check the soft theorem of $\mathcal{N}=8$ SUGRA amplitudes by some examples of the MHV sector. We mainly focus on the leading and the sub-leading soft gravitino divergences and the leading soft 
gravi-photon divergence, as well as the property of amplitudes with soft scalar.

First of all, we analyse a special class of amplitudes which are proportional to MHV amplitudes of gravitons. For such amplitudes, we can check soft gravitino divergences or soft gravi-photon divergences by using only soft graviton theorem, Eq. (2.2) or Eq. (4.17). In MHV sector of $\mathcal{N}=8$ SUGRA, there exists the following supersymmetry Ward identities: ${ }^{4}$

$$
\begin{aligned}
& \mathcal{M}_{n+1}\left(h_{1}^{-}, \psi_{2}^{A}, h_{3}^{+}, \ldots, h_{n}^{+}, \psi_{s B}\right) \\
& \quad=\delta_{B}^{A} \frac{\langle 1, s\rangle}{\langle 1,2\rangle} \mathcal{M}_{n+1}\left(h_{1}^{-}, h_{2}^{-}, h_{3}^{+}, \ldots, h_{n}^{+}, h_{s}^{+}\right), \\
& \mathcal{M}_{n+1}\left(h_{1}^{-}, v_{2}^{A B}, h_{3}^{+}, \ldots, h_{n}^{+}, v_{s C D}\right) \\
& \quad=\delta_{C D}^{A B} \frac{\langle 1, s\rangle^{2}}{\langle 1,2\rangle^{2}} \mathcal{M}_{n+1}\left(h_{1}^{-}, h_{2}^{-}, h_{3}^{+}, \ldots, h_{n}^{+}, h_{s}^{+}\right), \\
& \mathcal{M}_{n+1}\left(h_{1}^{-}, \chi_{2}^{A B C}, h_{3}^{+}, \ldots, h_{n}^{+}, \chi_{s D E F}\right) \\
& \quad=\delta_{D E F}^{A B C} \frac{\langle 1, s\rangle^{3}}{\langle 1,2\rangle^{3}} \mathcal{M}_{n+1}\left(h_{1}^{-}, h_{2}^{-}, h_{3}^{+}, \ldots, h_{n}^{+}, h_{s}^{+}\right), \\
& \mathcal{M}_{n+1}\left(h_{1}^{-}, S_{2}^{A B C D}, h_{3}^{+}, \ldots, h_{n}^{+}, S_{S E F G H}\right) \\
& =\delta_{E F G H}^{A B C D} \frac{\langle 1, s\rangle^{4}}{\langle 1,2\rangle^{4}} \mathcal{M}_{n+1}\left(h_{1}^{-}, h_{2}^{-}, h_{3}^{+}, \ldots, h_{n}^{+}, h_{s}^{+}\right)
\end{aligned}
$$

where $S^{A B C D}=\frac{1}{4 !} \epsilon^{A B C D E F G H} S_{E F G H}$. With the help of these Ward identities, we can study soft divergences of amplitudes involving lower-spinor soft particles by using only soft graviton theorem.

\subsubsection{Soft gravitino}

Here we study the soft gravitino divergence. For the right hand side of SWI (4.22), by using the soft graviton theorem we have

$$
\begin{aligned}
\delta_{B}^{A} & \frac{\langle 1, s\rangle}{\langle 1,2\rangle}\left(\frac{1}{\epsilon^{2}} S^{(0)}+\frac{1}{\epsilon} S^{(1)}\right) \mathcal{M}_{n}\left(h_{1}^{-}, h_{2}^{-}, h_{3}^{+}, \ldots, h_{n}^{+}\right) \\
& +\mathcal{O}\left(\epsilon^{0}\right) .
\end{aligned}
$$

First we consider the leading order $\mathcal{O}\left(\epsilon^{-2}\right)$ :

$$
\begin{aligned}
& \delta_{B}^{A} \frac{1}{\epsilon^{2}} \frac{\langle 1, s\rangle}{\langle 1,2\rangle} S^{(0)} \mathcal{M}_{n}\left(h_{1}^{-}, h_{2}^{-}, h_{3}^{+}, \ldots, h_{n}^{+}\right) \\
& \quad=\delta_{B}^{A} \frac{1}{\epsilon^{2}} \sum_{a=2}^{n-1} \frac{\langle 1, a\rangle}{\langle 1,2\rangle} \frac{[s, a]\langle n, a\rangle}{\langle s, a\rangle\langle n, s\rangle} \mathcal{M}_{n}\left(h_{1}^{-}, h_{2}^{-}, h_{3}^{+}, \ldots, h_{n}^{+}\right) .
\end{aligned}
$$

${ }^{4}$ Here the generalized Kronecker delta-symbol is defined as

$\delta_{B_{1} \ldots B_{n}}^{A_{1} \ldots A_{n}} \equiv \sum_{\sigma \in S_{n}} \operatorname{sgn}(\sigma) \delta_{B_{1}}^{A_{\sigma(1)}} \cdots \delta_{B_{n}}^{A_{\sigma(n)}}$.
In order to get the sub-leading order, substituting soft operator $S^{(1)}$ into the right hand side of Eq. (4.26), we have

$$
\begin{aligned}
\delta_{B}^{A} & \frac{1}{\epsilon} \frac{\langle 1, s\rangle}{\langle 1,2\rangle} S^{(1)} \mathcal{M}_{n}\left(h_{1}^{-}, h_{2}^{-}, h_{3}^{+}, \ldots, h_{n}^{+}\right) \\
\quad & \delta_{B}^{A} \frac{1}{\epsilon} \sum_{a=2}^{n} \frac{[s, a]}{\langle s, a\rangle} \frac{\langle 1, a\rangle}{\langle 1,2\rangle} \tilde{\lambda}_{s \dot{\alpha}} \frac{\partial}{\partial \tilde{\lambda}_{a \dot{\alpha}}} \mathcal{M}_{n}\left(h_{1}^{-}, h_{2}^{-}, h_{3}^{+}, \ldots, h_{n}^{+}\right) .
\end{aligned}
$$

Here the gauge freedom of $S^{(0)}$ is fixed by taking $x=y=1$.

Next we study the soft-gravitino divergence by using directly the soft gravitino theorem (4.18). From the left hand side of the identity (4.22), using the leading soft gravitino theorem (4.18), one can obtain:

$$
\begin{aligned}
& \frac{1}{\epsilon^{2}} \sum_{a=1}^{n-1} \frac{[s, a]\langle n, a\rangle}{\langle s, a\rangle\langle n, s\rangle} \mathcal{M}_{n}\left(h_{1}^{-}, \psi_{2}^{A}, \ldots, \mathscr{Q}_{a B} \Phi_{a}, \ldots, h_{n}^{+}\right) \\
& =\frac{1}{\epsilon^{2}}\left(\frac{[s, 2]\langle n, 2\rangle}{\langle s, 2\rangle\langle n, s\rangle} \mathcal{M}_{n}\left(h_{1}^{-}, h_{2}^{-}, \ldots, h_{n}^{+}\right) \delta_{B}^{A}\right. \\
& \left.\quad+\sum_{a=3}^{n-1} \frac{[s, a]\langle n, a\rangle}{\langle s, a\rangle\langle n, s\rangle} \mathcal{M}_{n}\left(h_{1}^{-}, \psi_{2}^{A}, \ldots, \psi_{a B}, \ldots, h_{n}^{+}\right)\right) \\
& =\delta_{B}^{A} \frac{1}{\epsilon^{2}} \sum_{a=2}^{n-1} \frac{[s, a]\langle n, a\rangle}{\langle s, a\rangle\langle n, s\rangle} \frac{\langle 1, a\rangle}{\langle 1,2\rangle} \mathcal{M}_{n}\left(h_{1}^{-}, h_{2}^{-}, h_{3}^{+}, \ldots, h_{n}^{+}\right) .
\end{aligned}
$$

This gives the same result as Eq. (4.27). Here one has used the SUSY Ward identity (4.22) and the operator $\mathscr{Q}_{a A}$ is defined by

$$
\begin{aligned}
& \mathscr{Q}_{a A} h_{a}^{+} \equiv \psi_{a A}, \mathscr{Q}_{a A} \psi_{a B} \equiv v_{a A B}, \\
& \mathscr{Q}_{a A} \psi_{a}^{B} \equiv \delta_{A}^{B} h_{a}^{-}, \mathscr{Q}_{a A} h_{a}^{-} \equiv 0, \ldots
\end{aligned}
$$

Roughly speaking, it make spin (or helicity) of the particle reduce by one half when an operator $\mathscr{Q}_{a A}$ act on this particle. Similarly, applying straightforwardly the sub-leading soft gravitino theorem (4.18) to the left hand side of the identity (4.22) gives

$$
\begin{aligned}
& \frac{1}{\epsilon} \sum_{a=1}^{n} \frac{[s, a]}{\langle s, a\rangle} \tilde{\lambda}_{s \dot{\alpha}} \frac{\partial}{\partial \tilde{\lambda}_{a \dot{\alpha}}} \mathcal{M}_{n}\left(h_{1}^{-}, \psi_{2}^{A}, \ldots, \mathscr{Q}_{a B} \Phi_{a}, \ldots, h_{n}^{+}\right) \\
& \quad=\delta_{B}^{A} \frac{1}{\epsilon} \sum_{a=2}^{n} \frac{[s, a]}{\langle s, a\rangle} \frac{\langle 1, a\rangle}{\langle 1,2\rangle} \tilde{\lambda}_{s \dot{\alpha}} \frac{\partial}{\partial \tilde{\lambda}_{a \dot{\alpha}}} \mathcal{M}_{n}\left(h_{1}^{-}, h_{2}^{-}, h_{3}^{+}, \ldots, h_{n}^{+}\right) .
\end{aligned}
$$

This give the same result as the one from the soft graviton theorem, Eq. (4.28).

\subsubsection{Soft gravi-photon}

Since there is only leading soft gravi-photon divergence, just Weinberg's leading soft graviton theorem is need. In the holo- 
morphic soft limit $\lambda_{s} \rightarrow \epsilon \lambda_{s}$, using leading soft graviton theorem ${ }^{5}$ the right hand side of the SWI (4.23) becomes

$$
\begin{aligned}
\frac{1}{\epsilon} \delta_{C D}^{A B} \frac{\langle 1, s\rangle^{2}}{\langle 1,2\rangle^{2}} S^{(0)} \mathcal{M}_{n}\left(h_{1}^{-}, h_{2}^{-}, h_{3}^{+}, \ldots, h_{n}^{+}\right)+\mathcal{O}\left(\epsilon^{0}\right) \\
=\frac{1}{\epsilon} \delta_{C D}^{A B} \sum_{a=2}^{n} \frac{\langle 1, a\rangle^{2}}{\langle 1,2\rangle^{2}} \frac{[s, a]}{\langle s, a\rangle} \\
\quad \times \mathcal{M}_{n}\left(h_{1}^{-}, h_{2}^{-}, h_{3}^{+}, \ldots, h_{n}^{+}\right)+\mathcal{O}\left(\epsilon^{0}\right)
\end{aligned}
$$

On the other hand, by applying straightforwardly the soft gravi-photon theorem (4.19) to the left hand side of the SWI (4.23), one gets

$$
\begin{aligned}
\mathcal{M}_{n+1}\left(h_{1}^{-}, v_{2}^{A B}, \ldots, v_{s C D}\right) \\
=\frac{1}{\epsilon} \sum_{a=1}^{n} \frac{[s, a]}{\langle s, a\rangle} \mathcal{M}_{n}\left(h_{1}^{-}, v_{2}^{A B-}, \ldots, \mathscr{Q}_{a D} \mathscr{Q}_{a C} \Phi_{a}, \ldots, h_{n}^{+}\right) \\
\quad+\mathcal{O}\left(\epsilon^{0}\right) \\
=\frac{1}{\epsilon}\left(\frac{[s, 2]}{\langle s, 2\rangle} \mathcal{M}_{n}\left(h_{1}^{-}, h_{2}^{-}, \ldots, h_{n}^{+}\right) \delta_{C D}^{A B}\right. \\
\left.\quad+\sum_{a=3}^{n} \frac{[s, a]}{\langle s, a\rangle} \mathcal{M}_{n}\left(h_{1}^{-}, v_{2}^{A B}, \ldots, v_{a C D}, \ldots, h_{n}^{+}\right)\right)+\mathcal{O}\left(\epsilon^{0}\right) \\
=\frac{1}{\epsilon} \delta_{C D}^{A B} \sum_{a=2}^{n} \frac{[s, a]}{\langle s, a\rangle} \frac{\langle 1, a\rangle^{2}}{\langle 1,2\rangle^{2}} \mathcal{M}_{n}\left(h_{1}^{-}, h_{2}^{-}, h_{3}^{+}, \ldots, h_{n}^{+}\right) \\
+\mathcal{O}\left(\epsilon^{0}\right) .
\end{aligned}
$$

Here one has used the Ward identity (4.23).

Next we analyse two 4-point amplitudes which have been computed by using both generating function method proposed in [58] and Feynman diagrams respectively in [62]. The first example is 4-gravi-photon amplitude:

$$
\begin{aligned}
& \mathcal{M}_{4}\left(v^{A B}, v^{C D}, v_{E F}, v_{G H}\right) \\
& =\langle 12\rangle^{2}[34]^{2}\left(\frac{1}{t} \delta_{E F}^{A B} \delta_{G H}^{C D}+\frac{1}{u} \delta_{G H}^{A B} \delta_{E F}^{C D}+\frac{1}{s} \delta_{E F G H}^{A B C D}\right)
\end{aligned}
$$

where $s, t, u$ are Mandelstam variables

$$
\begin{aligned}
& s=\left(k_{1}+k_{2}\right)^{2}=\left(k_{3}+k_{4}\right)^{2}=s_{12}=s_{34}, \\
& t=\left(k_{1}+k_{3}\right)^{2}=\left(k_{2}+k_{4}\right)^{2}=s_{13}=s_{24}, \\
& u=\left(k_{1}+k_{4}\right)^{2}=\left(k_{2}+k_{3}\right)^{2}=s_{14}=s_{23} .
\end{aligned}
$$

In holomorphic soft limit $\lambda_{4} \rightarrow \epsilon \lambda_{4}$, the amplitude becomes

$$
\begin{aligned}
& \mathcal{M}_{4}\left(v^{A B}, v^{C D}, v_{E F}, \epsilon v_{G H}\right) \\
& =\frac{1}{\epsilon}\left(\frac{\langle 12\rangle^{2}[34]^{2}}{\langle 24\rangle[24]} \delta_{E F}^{A B} \delta_{G H}^{C D}+\frac{\langle 12\rangle^{2}[34]^{2}}{\langle 14\rangle[14]} \delta_{G H}^{A B} \delta_{E F}^{C D}\right.
\end{aligned}
$$

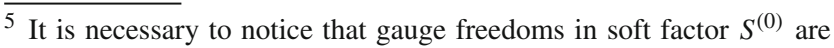
fixed by setting $x=y=1$.

$$
\begin{aligned}
& \left.+\frac{\langle 12\rangle^{2}[34]^{2}}{\langle 34\rangle[34]} \delta_{E F G H}^{A B C D}\right) \\
= & \frac{1}{\epsilon}\left(\frac{[24]}{\langle 24\rangle} \frac{\langle 12\rangle^{4}}{\langle 13\rangle^{2}} \delta_{E F}^{A B} \delta_{G H}^{C D}+\frac{[14]}{\langle 14\rangle} \frac{\langle 12\rangle^{4}}{\langle 23\rangle^{2}} \delta_{G H}^{A B} \delta_{E F}^{C D}\right. \\
& \left.+\frac{[34]\langle 12\rangle^{2}}{\langle 34\rangle} \delta_{E F G H}^{A B C D}\right)
\end{aligned}
$$

which is exact in $\epsilon$. In the last line, one used the momentum conservation conditions: $\langle 12\rangle[24]=-\langle 13\rangle[34]$ and $\langle 21\rangle[14]=-\langle 23\rangle[34]$.

Notice that following 3-point amplitudes of $\mathcal{N}=8$ SUGRA:

$$
\begin{aligned}
\mathcal{M}_{3}\left(h_{1}^{-}, v_{2}^{C D}, v_{3 E F}\right) & =\frac{\langle 12\rangle^{4}}{\langle 23\rangle^{2}} \delta_{E F}^{C D}, \\
\mathcal{M}_{3}\left(v_{1}^{A B}, h_{2}^{-}, v_{3 E F}\right) & =\frac{\langle 12\rangle^{4}}{\langle 31\rangle^{2}} \delta_{E F}^{A B}, \\
\mathcal{M}_{3}\left(v_{1}^{A B}, v_{2}^{C D}, S_{3 E F G H}\right) & =\langle 12\rangle^{2} \delta_{E F G H}^{A B C D} .
\end{aligned}
$$

Then one finds that

$$
\begin{aligned}
\mathcal{M}_{4}\left(v^{A B}, v^{C D}, v_{E F}, \epsilon v_{G H}\right) \\
=\frac{1}{\epsilon}\left(\frac{[24]}{\langle 24\rangle} \mathcal{M}_{3}\left(v_{1}^{A B}, h_{2}^{-}, v_{3 E F}\right) \delta_{G H}^{C D}\right. \\
+\frac{[14]}{\langle 14\rangle} \mathcal{M}_{3}\left(h_{1}^{-}, v_{2}^{C D}, v_{3 E F}\right) \delta_{G H}^{A B} \\
\left.+\frac{[34]}{\langle 34\rangle} \mathcal{M}_{3}\left(v_{1}^{A B}, v_{2}^{C D}, S_{3 E F G H}\right)\right) .
\end{aligned}
$$

Very nice! This is just the result from soft gravi-photon theorem.

Another example is 2-scalar 2-gravi-photon amplitude:

$$
\begin{aligned}
& \mathcal{M}_{4}\left(v^{A B}, v_{C D}, S_{E F G H}, S_{I J K L}\right) \\
& =\langle 13\rangle^{2}[23]^{2}\left(\frac{1}{s} \delta_{C D}^{A B} \epsilon_{E F G H I J K L}\right. \\
& \left.+\frac{3 !}{t} \delta_{[E F}^{A B} \epsilon_{G H] I J K L C D}+\frac{3 !}{u} \delta_{[I J}^{A B} \epsilon_{K L] E F G H C D}\right) .
\end{aligned}
$$

We study the property of this amplitude when external particle $v_{C D}$ becomes soft. In holomorphic soft limit $\lambda_{2} \rightarrow \epsilon \lambda_{2}$, this amplitude becomes

$$
\begin{aligned}
& \mathcal{M}_{4}\left(v^{A B}, \epsilon v_{C D}, S_{E F G H}, S_{I J K L}\right) \\
& =\frac{1}{\epsilon}\left(\frac{[12]}{\langle 12\rangle} \frac{\langle 13\rangle^{2}\langle 14\rangle^{2}}{\langle 34\rangle^{2}} \delta_{C D}^{A B} \epsilon_{E F G H I J K L}\right. \\
& +3 ! \frac{[24]}{\langle 24\rangle}\langle 14\rangle^{2} \delta_{[E F}^{A B} \epsilon_{G H] I J K L C D} \\
& \left.+3 ! \frac{[23]}{\langle 23\rangle}\langle 13\rangle^{2} \delta_{[I J}^{A B} \epsilon_{K L] E F G H C D}\right) .
\end{aligned}
$$


Here used the momentum conservation conditions: $\frac{[23]}{[12]}=$ $\frac{\langle 14\rangle}{\langle 34\rangle}$ and $\langle 13\rangle[23]=-\langle 14\rangle[24]$.

Note the following three-point MHV amplitudes:

$\mathcal{M}_{3}\left(h_{1}^{-}, S_{3 E F G H}, S_{4 I J K L}\right)=\frac{\langle 13\rangle^{2}\langle 14\rangle^{2}}{\langle 34\rangle^{2}} \epsilon_{E F G H I J K L}$

$\mathcal{M}_{3}\left(v_{1}^{A B}, v_{3}^{M N}, S_{4 I J K L}\right)=\langle 13\rangle^{2} \delta_{I J K L}^{A B M N}$

$\mathcal{M}_{3}\left(v_{1}^{A B}, S_{3 E F G H}, v_{4}^{M N}\right)=\langle 14\rangle^{2} \delta_{E F G H}^{A B M N}$

Then we have 6

$$
\begin{aligned}
& \mathcal{M}_{4}\left(v^{A B}, \epsilon v_{C D}, S_{E F G H}, S_{I J K L}\right) \\
& =\frac{1}{\epsilon}\left(\frac{[21]}{\langle 21\rangle} \delta_{C D}^{A B} \mathcal{M}_{3}\left(h_{1}^{-}, S_{3 E F G H}, S_{4 I J K L}\right)\right. \\
& +\frac{1}{2} \frac{[23]}{\langle 23\rangle} \epsilon_{M N E F G H C D} \mathcal{M}_{3}\left(v_{1}^{A B}, v_{3}^{M N}, S_{4 I J K L}\right) \\
& \left.+\frac{1}{2} \frac{[24]}{\langle 24\rangle} \epsilon_{M N I J K L C D} \mathcal{M}_{3}\left(v_{1}^{A B}, S_{3 E F G H}, v_{4}^{M N}\right)\right) .
\end{aligned}
$$

By applying straightforwardly the soft gravi-photon theorem (4.19) to amplitude $\mathcal{M}_{4}\left(v^{A B}, v_{C D}, S_{E F G H}, S_{I J K L}\right)$, one can also obtain the same result.

\subsubsection{Soft gravi-photino and soft scalar}

According to SUSY Ward identities (4.24) and (4.25), one finds

$$
\begin{aligned}
& \mathcal{M}_{n+1}\left(h_{1}^{-}, \chi_{2}^{A B C}, h_{3}^{+}, \ldots, h_{n}^{+}, \chi_{s D E F}\right) \sim \mathcal{O}\left(\epsilon^{0}\right), \\
& \mathcal{M}_{n+1}\left(h_{1}^{-}, S_{2}^{A B C D}, h_{3}^{+}, \ldots, h_{n}^{+}, S_{S E F G H}\right) \sim \mathcal{O}(\epsilon)
\end{aligned}
$$

in the holomorphic soft limit $\lambda_{s} \rightarrow \epsilon \lambda_{s}$. These results accord with the soft theorems (4.20) and (4.21).

Next we study the 2-scalar 2-gravi-photon amplitude (4.43) which have been discussed previously. In the holomorphic soft limit $\lambda_{4} \rightarrow \epsilon \lambda_{4}$, since $\langle 13\rangle^{2}[23]^{2}=\langle 14\rangle^{2}[24]^{2} \sim$ $\epsilon^{2}, s \sim t \sim u \sim \epsilon$, this amplitude becomes

$\left.\mathcal{M}_{4}\left(v^{-} v^{+} \phi \phi\right)\right|_{\lambda_{4} \rightarrow \epsilon \lambda_{4}} \sim \epsilon \rightarrow 0$.

In the MHV sector of $\mathcal{N}=8$ SUGRA, there are also amplitudes involving four scalars. Here we analyse a 4-scalar amplitude which was computed in [62]:

\footnotetext{
${ }^{6}$ Here one has used a identity

$\frac{1}{2} \delta_{E F G H}^{A B M N} \epsilon_{M N I J K L C D}=3 ! \delta_{[E F}^{A B} \epsilon_{G H] I J K L C D}$.
}

$$
\begin{aligned}
\mathcal{M}_{4} & \left(S_{A B C D}, S_{E F G H}, S_{I J K L}, S_{M N P Q}\right) \\
= & \frac{t u}{s} \epsilon_{A B C D E F G H} \epsilon_{I J K L M N P Q} \\
& +\frac{s u}{t} \epsilon_{A B C D I J K L} \epsilon_{E F G H M N P Q} \\
& +\frac{s t}{u} \epsilon_{A B C D M N P Q} \epsilon_{E F G H I J K L} \\
& +\frac{1}{2(4 !)^{3}} \sum_{\sigma} \operatorname{sgn}(\sigma)\left[s \epsilon_{1} 1_{2} 1_{3} 1_{4} 3_{1} 3_{2} 4_{3} 4_{4} \epsilon_{2} 2_{2} 2_{3} 2_{4} 4_{1} 4_{2} 3_{3} 3_{4}\right. \\
& +t \epsilon_{1_{1} 1_{2} 1_{3} 1_{4} 2_{1} 2_{2} 4_{3} 4_{4}} \epsilon_{3} 3_{2} 3_{3} 3_{4} 4_{1} 4_{2} 2_{3} 2_{4} \\
& \left.+u \epsilon_{1_{1} 1_{2} 1_{3} 1_{4} 2_{1} 2_{2} 3_{3} 3_{4}} \epsilon_{4} 4_{2} 4_{3} 4_{4} 3_{1} 3_{2} 2_{3} 2_{4}\right] .
\end{aligned}
$$

Here $\left\{1_{1} 1_{2} 1_{3} 1_{4}\right\}$ denotes permutations of $\{A, B, C, D\}$ and so forth [62]. In holomorphic soft limit $\lambda_{4} \rightarrow \epsilon \lambda_{4}$, since $s \sim t \sim u \sim \epsilon$, the amplitude behaves as

$\left.\mathcal{M}_{4}(\phi \phi \phi \phi)\right|_{\lambda_{4} \rightarrow \epsilon \lambda_{4}} \sim \epsilon \rightarrow 0$

A great deal of research shows that amplitudes vanish in soft scalar limit, which indicates a hidden global $E_{7(7)}$ symmetry of classical $\mathcal{N}=8$ SUGRA [56,58,85-90]. This is consist with our soft theorem (4.21).

\subsection{SUGRA soft operators as double copy of SYM soft operators}

As discussed in Sect. 2.3, the gravity soft operator can be expressed as double copy of gauge theory soft operators. This also occurs in supersymmetric theories. In the end of this section, we write the soft operators in $\mathcal{N}=8$ SUGRA in terms of a sum of some products of soft operators in $\mathcal{N}=4$ SYM.

First introducing a new operator involving the derivative with respect to Grassmann odd variable $\eta_{a}^{A}$ as follows:

$\mathfrak{S}_{\eta}^{1}(s, a) \equiv \frac{1}{\langle s, a\rangle} \eta_{s}^{A} \frac{\partial}{\partial \eta_{a}^{A}}$

Then the soft operators in $\mathcal{N}=4$ SYM may be written as

$\mathcal{S}_{\mathrm{SYM}}^{(0)}=S_{\mathrm{YM}}^{(0)}=\mathfrak{S}^{0}(x, s, n)+\mathfrak{S}^{0}(x, s, 1)$,

$\mathcal{S}_{\mathrm{SYM}}^{(1)}=\left(\mathfrak{S}^{1}(s, 1)-\mathfrak{S}^{1}(s, n)\right)+\left(\mathfrak{S}_{\eta}^{1}(s, 1)-\mathfrak{S}_{\eta}^{1}(s, n)\right)$.

The 'KLT-like formula' of the leading soft factor $\mathcal{S}^{(0)}$ in gravity has been obtained in Sect. 2.3. The sub-leading soft operator may be written as:

$$
\begin{aligned}
\mathcal{S}^{(1)}= & \frac{1}{2} \sum_{a=1}^{n} s_{s a}\left(\mathfrak{S}^{0}(x, s, a)+\mathfrak{S}^{0}(y, s, a)\right) \\
& \times\left(\mathfrak{S}^{1}(s, a)+\mathfrak{S}_{\eta}^{1}(s, a)\right)
\end{aligned}
$$


where $\lambda_{x}$ and $\lambda_{y}$ are arbitrary reference spinors. The subsub-leading soft operator may be expressed as

$$
\begin{aligned}
\mathcal{S}^{(2)}= & \frac{1}{2} \sum_{a=1}^{n} s_{s a}\left(\mathfrak{S}^{1}(s, a)+\mathfrak{S}_{\eta}^{1}(s, a)\right) \\
& \times\left(\mathfrak{S}^{1}(s, a)+\mathfrak{S}_{\eta}^{1}(s, a)\right) .
\end{aligned}
$$

As mentioned in Sect. 2.3, all derivatives in operators only act on amplitudes. These relations may be derived using the scheme proposed in [19] by super-KLT relation [72,76-78, 91,92].

\section{Conclusion and discussions}

In this work, the super soft theorems were investigated systematically in 4D maximally $\mathcal{N}=4$ super-Yang-Mills theory and $\mathcal{N}=8$ supergravity. We have presented the super soft theorems with rigorous proofs at tree level. The main results are Eq. (3.19) for SYM and Eq. (4.9) for SUGRA. In $\mathcal{N}=4 \mathrm{SYM}$, several simple examples were examined and the results were in agreement with the super soft theorem exactly. In $\mathcal{N}=8$ SUGRA, employing the SUSY Ward identities, we verified the soft gravitino and soft gravi-photon divergences by using leading and sub-leading soft graviton theorem in the MHV sector. Several four-point amplitudes were also checked in details.

There are several further topics that are fascinating for us. First, properties of amplitudes involving soft fermion should be investigated more systematically. In this paper, we discussed the soft gluino divergence for color-ordered amplitudes of $\mathcal{N}=4 \mathrm{SYM}$ and the soft gravitino divergence and the soft gravi-photino divergence for $\mathcal{N}=8$ SUGRA amplitudes. It will be interesting to study the properties of amplitudes involving soft fermion in other theories.

Second, it will be interesting to find other methods to derive the soft theorems. Let us take an example. In [57], all tree-level superamplitudes in $\mathcal{N}=4 \mathrm{SYM}$ were expressed as compact analytical formulas. By taking the soft limit directly, it gives the soft theorem as shown in Appendix A. The similar formulas for all tree-level superamplitudes in $\mathcal{N}=8$ SUGRA were also obtained in [60]. We will also study soft theorem through these formulas in future work.

Finally, more on the relations between the soft theorems and symmetry principle should be understood.

Although the leading and sub-leading soft graviton theorems in gravity [25-27,32,93] and leading and sub-leading soft-photon theorems in massless QED [17] were interpreted as symmetries of $\mathcal{S}$-matrixes in recent works, very limited information was known for other soft divergences. Our particular interest is to explore the remarkable relations between super soft theorems and local supersymmetry.
Acknowledgments The author would like to thank Professor Jun-Bao $\mathrm{Wu}$ for suggesting this project as well as numerous discussions and valuable comments through out all the stages of the work. He would also especially like to thank Professor Chuan-Jie Zhu for his support, guidance, discussions and careful reading of the manuscript. He is also grateful to Da-Ping Liu, Wen-Jian Pan, Yu Tian, Gang Yang and Hongbao Zhang for various helpful discussions. This work was supported by the National Natural Science Foundation of China under Grants No. 11135006.

Open Access This article is distributed under the terms of the Creative Commons Attribution License which permits any use, distribution, and reproduction in any medium, provided the original author(s) and the source are credited.

Funded by $\mathrm{SCOAP}^{3}$ / License Version CC BY 4.0.

\section{Appendix A: Alternative derivation of soft theorem in $\mathcal{N}=4 \mathrm{SYM}$}

In this appendix, we rederive the soft theorem of $\mathcal{N}=4$ SYM by using formulaes for all tree-level superamplitudes which were given in [57].

First of all, we have to summarize briefly main results of Drummond and Henn's paper [57]. Three-point amplitudes are fundamental in BCFW-construction of higher-point amplitudes. In Sect. 3, three-point MHV and Googly (or antiMHV) superamplitudes of $\mathcal{N}=4 \mathrm{SYM}$ have been presented. By solving super-BCFW resursion, it is easy to obtain $n$-point $(n>3)$ MHV superamplitudes

$\mathscr{A}_{n}^{\mathrm{MHV}}\left(\left\{\lambda_{a}, \tilde{\lambda}_{a}, \eta_{a}\right\}\right)=\frac{\delta^{4}(p) \delta^{8}(q)}{\langle 12\rangle\langle 23\rangle \cdots\langle n 1\rangle}$.

The $n$-point MHV superamplitude is simple and compact, just as Parke-Taylor formula of the pure gluonic amplitude. The delta functions $\delta^{4}(p)$ and $\delta^{8}(q)$ are consequences of translation invariance and supersymmetry. Therefore all treelevel superamplitudes, not just MHV, contain delta function factor $\delta^{4}(p) \delta^{8}(q)$ in $\mathcal{N}=4 \mathrm{SYM}$. So it is very convenient to factor out the MHV superamplitude,

$\mathscr{A}_{n}=\mathscr{A}_{n}^{\mathrm{MHV}} \mathcal{P}_{n}$.

Here $\mathcal{P}_{n}$ is a function of spinors $\lambda_{a}, \tilde{\lambda}_{a}$ and Grassmann variables $\eta_{a}^{A}$ and one can express this quantity as following form:

$\mathcal{P}_{n}=\mathcal{P}_{n}^{\mathrm{MHV}}+\mathcal{P}_{n}^{\mathrm{NMHV}}+\cdots+\mathcal{P}_{n}^{\overline{\mathrm{MHV}}}$.

Of course $\mathcal{P}_{n}^{\mathrm{MHV}}=1$, and the $\mathrm{N}^{k} \mathrm{MHV}$ function $\mathcal{P}_{n}^{\mathrm{N}^{k} \mathrm{MHV}}$ has Grassmann degree $4 k$.

Turning to the NMHV sector, the function $\mathcal{P}_{n}^{\mathrm{NMHV}}$ is given by [57]

$\mathcal{P}_{n}^{\mathrm{NMHV}}=\sum_{2 \leq a<b \leq n-1} R_{n ; a b}$.

Here $R_{n ; a b}$ is a dual superconformal invariant [46-51,57-60] 


$$
\begin{aligned}
& R_{n ; a b} \\
& =\frac{\langle a, a-1\rangle\langle b, b-1\rangle \delta^{4}\left(\Xi_{n ; a b}\right)}{x_{a b}^{2}\left\langle n\left|x_{n a} x_{a b}\right| b\right\rangle\left\langle n\left|x_{n a} x_{a b}\right| b-1\right\rangle\left\langle n\left|x_{n b} x_{b a}\right| a\right\rangle\left\langle n\left|x_{n b} x_{b a}\right| a-1\right\rangle}
\end{aligned}
$$

where

$$
\begin{aligned}
x_{i j} & \equiv k_{i}+k_{i+1}+\cdots+k_{j-1}, \\
\theta_{i j} & \equiv q_{i}+q_{i+1}+\cdots+q_{j-1}, \quad q_{a} \equiv \lambda_{a} \eta_{a},
\end{aligned}
$$

and the Grassmann odd quantity $\Xi_{n ; a b}$ is defined by

$$
\begin{aligned}
\Xi_{n ; a b} & \equiv\left\langle n\left|x_{n a} x_{a b}\right| \theta_{b n}\right\rangle+\left\langle n\left|x_{n b} x_{b a}\right| \theta_{a n}\right\rangle \\
& =\left\langlen \left|\left(x_{n a} x_{a b} \sum_{i=b}^{n-1}|i\rangle \eta_{i}+x_{n b} x_{b a} \sum_{i=a}^{n-1}|i\rangle \eta_{i}\right) .\right.\right.
\end{aligned}
$$

Obviously, this quantity is independent of $\eta_{1}$ and $\eta_{n}$. In fact, it is relevant to a special gauge choice.

Similarly, NNMHV function also may be constructed as follows

$$
\begin{aligned}
\mathcal{P}_{n}^{\mathrm{NNMHV}}= & \sum_{2 \leq a_{1}, b_{1} \leq n-1} R_{n, a_{1} b_{1}}^{0 ; 0} \\
& \times\left(\sum_{a_{1}+1 \leq a_{2}, b_{2} \leq b_{1}} R_{n ; b_{1} a_{1} ; a_{2} b_{2}}^{0 ; a_{1} b_{1}}+\sum_{b_{1} \leq a_{2}, b_{2} \leq n-1} R_{n ; a_{2} b_{2}}^{a_{1} b_{1} ; 0}\right) .
\end{aligned}
$$

Here has involved a quantity which is a generalization of the $R$-invariant,

$$
\begin{aligned}
& R_{n ; b_{1} a_{1} ; b_{2} a_{2} ; \ldots ; b_{r} a_{r} ; a b} \\
& =\frac{\langle a, a-1\rangle\langle b, b-1\rangle \delta^{4}\left(\Xi_{n ; b_{1} a_{1} ; b_{2} a_{2} ; \ldots ; b_{r} a_{r} ; a b}\right)}{x_{a b}^{2}|\xi\rangle x_{a_{r} a} x_{a b}|b\rangle|\xi\rangle x_{a_{r} a} x_{a b}|b-1\rangle|\xi\rangle x_{a_{r} b} x_{b a}|a\rangle|\xi\rangle x_{a_{r} b} x_{b a}|a-1\rangle} .
\end{aligned}
$$

For details, see [57].

More generally, all the $\mathcal{P}_{n}^{\mathrm{N}^{k} \mathrm{MHV}}$ functions can be written in

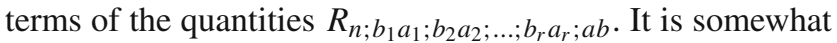
surprising that all quantities (A.10) are independent of $\eta_{1}$ and $\eta_{n}$ and so are all $\mathcal{P}_{n}^{\mathrm{N}^{k} \mathrm{MHV}}$, i.e.,

$\frac{\partial}{\partial \eta_{1}^{A}} \mathcal{P}_{n}=\frac{\partial}{\partial \eta_{n}^{A}} \mathcal{P}_{n}=0$.

In fact, this reflects the special gauge choice of shifted legs in $\mathrm{BCFW}$ recursion.

Now we turn to study soft theorem. The $n$-point MHV superamplitude is not only the simplest in all $n$-point amplitudes, but a common factor for all amplitudes. So we begin with MHV sectors.
First we write the delta function $\delta^{8}(q)$ as

$$
\begin{aligned}
\delta^{8}(q)= & \langle 1, n\rangle^{4} \delta^{4}\left(\frac{\langle n, s\rangle}{\langle n, 1\rangle} \eta_{s}^{A}+\eta_{1}^{A}+\sum_{a=2}^{n-1} \frac{\langle n, a\rangle}{\langle n, 1\rangle} \eta_{a}^{A}\right) \\
& \times \delta^{4}\left(\frac{\langle 1, s\rangle}{\langle 1, n\rangle} \eta_{s}^{B}+\eta_{n}^{B}+\sum_{a=2}^{n-1} \frac{\langle 1, a\rangle}{\langle 1, n\rangle} \eta_{a}^{B}\right) .
\end{aligned}
$$

When the soft particle is gluon, we have

$$
\begin{aligned}
& \mathscr{A}_{n+1}^{\mathrm{MHV}}\left(\Phi_{1}, \ldots, \Phi_{n}, g_{s}^{+}\right)=\frac{\delta^{4}(p)\langle 1, n\rangle^{4}}{\langle s, 1\rangle\langle 1,2\rangle \cdots\langle n, s\rangle} \\
& \quad \times \delta^{4}\left(\eta_{1}^{A}+\sum_{a=2}^{n-1} \frac{\langle n, a\rangle}{\langle n, 1\rangle} \eta_{a}^{A}\right) \delta^{4}\left(\eta_{n}^{B}+\sum_{a=2}^{n-1} \frac{\langle 1, a\rangle}{\langle 1, n\rangle} \eta_{a}^{B}\right) \\
& =\frac{\langle n, 1\rangle}{\langle s, 1\rangle\langle n, s\rangle} \frac{\delta^{4}(p)}{\langle 1,2\rangle \cdots\langle n, 1\rangle} \delta^{8}\left(\sum_{a=1}^{n} \lambda_{a}^{\alpha} \eta_{a}^{A}\right) .
\end{aligned}
$$

In the holomorphic soft limit $\lambda_{s} \rightarrow \epsilon \lambda_{s}$, it can give the leading soft factor of Yang-Mills amplitude.

When the soft particle is gluino, we have

$$
\begin{aligned}
\eta_{s}^{A} & \mathscr{A}_{n+1}^{\mathrm{MHV}}\left(\Phi_{1}, \ldots, \Phi_{n}, \psi_{s}\right) \\
= & \frac{\langle n, 1\rangle}{\langle n, s\rangle\langle s, 1\rangle} \frac{\delta^{4}(p)\langle 1, n\rangle^{4}}{\langle 1,2\rangle \cdots\langle n, 1\rangle} \\
& \times\left\{\frac{\langle n, s\rangle}{\langle n, 1\rangle} \times(-1)^{A+1} \eta_{s}^{A} \prod_{B \neq A}\left(\eta_{1}^{B}+\sum_{a=2}^{n-1} \frac{\langle n, a\rangle}{\langle n, 1\rangle} \eta_{a}^{B}\right)\right. \\
& \times \prod_{C}\left(\eta_{n}^{C}+\sum_{a=2}^{n-1} \frac{\langle 1, a\rangle}{\langle 1, n\rangle} \eta_{a}^{C}\right) \\
& +\frac{\langle 1, s\rangle}{\langle 1, n\rangle} \times(-1)^{A+1} \eta_{s}^{A} \prod_{C}\left(\eta_{1}^{C}+\sum_{a=2}^{n-1} \frac{\langle n, a\rangle}{\langle n, 1\rangle} \eta_{a}^{C}\right) \\
& \left.\times \prod_{B \neq A}\left(\eta_{n}^{B}+\sum_{a=2}^{n-1} \frac{\langle 1, a\rangle}{\langle 1, n\rangle} \eta_{a}^{B}\right)\right\} \\
= & \frac{\langle n, 1\rangle}{\langle n, s\rangle\langle s, 1\rangle}\left(\frac{\langle n, s\rangle}{\langle n, 1\rangle} \eta_{s}^{A} \frac{\partial}{\partial \eta_{1}^{A}}+\frac{\langle 1, s\rangle}{\langle 1, n\rangle} \eta_{s}^{A} \frac{\partial}{\partial \eta_{n}^{A}}\right) \\
& \times \mathscr{A}_{n}^{\mathrm{MHV}}\left(\Phi_{1}, \ldots, \Phi_{n}\right) .
\end{aligned}
$$

In the holomorphic soft limit $\lambda_{s} \rightarrow \epsilon \lambda_{s}$, it gives $\mathcal{O}\left(\epsilon^{-1}\right)$ order soft divergence. When the soft particle is scalar, it is also easy to see that MHV superamplitudes are invariant under the holomorphic soft rescaling $\lambda_{s} \rightarrow \epsilon \lambda_{s}$.

Another task is to see the function $\mathcal{P}_{n+1}$ in the holomorphic soft limit. First we consider soft behavior of NMHV function: 7

\footnotetext{
$\overline{7}$ Here we fix gauge such that the function $\mathcal{P}_{n+1}^{\mathrm{NMHV}}$ is independent of $\eta_{n}$ and $\eta_{s}$.
} 
$\mathcal{P}_{n+1}^{\mathrm{NMHV}}(1, \ldots, \hat{n}, \hat{s})=\sum_{1 \leq a<b \leq n-1} R_{n ; a b}(1, \ldots, \hat{n}, \hat{s})$

Here dual superconformal invariant $R_{n ; a b}$ is given by

$$
\begin{aligned}
& R_{n ; a b}(1, \ldots, \hat{n}, \hat{s}) \\
& \quad=\frac{\langle a, a-1\rangle\langle b, b-1\rangle \delta^{4}\left(\Xi_{n ; a b}\right)}{\tilde{x}_{a b}^{2}\left\langle n\left|\tilde{x}_{n a} \tilde{x}_{a b}\right| b\right\rangle\left\langle n\left|\tilde{x}_{n a} \tilde{x}_{a b}\right| b-1\right\rangle\left\langle n\left|\tilde{x}_{n b} \tilde{x}_{b a}\right| a\right\rangle\left\langle n\left|\tilde{x}_{n b} \tilde{x}_{b a}\right| b-1\right\rangle}
\end{aligned}
$$

where

$$
\Xi_{n ; a b}=\left\langlen \left|\left(\tilde{x}_{n a} \tilde{x}_{a b} \sum_{i=b}^{n-1}|i\rangle \eta_{i}+\tilde{x}_{n b} \tilde{x}_{b a} \sum_{i=a}^{n-1}|i\rangle \eta_{i}\right) .\right.\right.
$$

Here the dual variable $\tilde{x}_{a b}$ corresponds to the ordering $(1, \ldots, n, s)$ of $(n+1)$-point color-ordered superamplitude $\mathscr{A}_{n+1}\left(\Phi_{1}, \ldots, \Phi_{n}, \Phi_{s}\right)$ while the variable $x_{a b}$ corresponds to the ordering $(1, \ldots, n)$ of $n$-point superamplitude $\mathscr{A}_{n}\left(\Phi_{1}, \ldots, \Phi_{n}\right)$.

In the holomorphic soft limit $\lambda_{s} \rightarrow \epsilon \lambda_{s}$,

$$
\begin{aligned}
\langle a, a-1\rangle & = \begin{cases}\epsilon\langle 1, s\rangle & \text { for } a=1, \\
\langle a, a-1\rangle & \text { for } a \geq 2,\end{cases} \\
\tilde{x}_{n a} & =\epsilon k_{s}+k_{1}+\cdots+k_{a-1}=x_{n a}+\epsilon k_{s}, \\
\tilde{x}_{a b} & =x_{a b} \text { for } 1 \leq a<b \leq n-1, \\
\tilde{x}_{b a} & =x_{b a}+\epsilon k_{s} \text { for } 1 \leq a<b \leq n-1, \\
\frac{1}{\tilde{x}_{n a}} & =\frac{1}{x_{n a}}+\mathcal{O}(\epsilon), \\
\frac{1}{\tilde{x}_{b a}} & =\frac{1}{x_{b a}}+\mathcal{O}(\epsilon) \text { for } 1 \leq a<b \leq n-1 .
\end{aligned}
$$

Substituting above formulas into Eq. (A.15), one finds

$$
\begin{gathered}
\mathcal{P}_{n+1}^{\mathrm{NMHV}}(1, \ldots, \hat{n}, \hat{s})=\sum_{2 \leq a<b \leq n-1} R_{n ; a b}(\hat{1}, \ldots, \hat{n})+\mathcal{O}(\epsilon) \\
=\mathcal{P}_{n}^{\mathrm{NMHV}}(\hat{1}, \ldots, \hat{n})+\mathcal{O}(\epsilon) .
\end{gathered}
$$

in the holomorphic soft limit $\lambda_{s} \rightarrow \epsilon \lambda_{s}$. Applying same analysis to $\mathcal{P}_{n}^{\mathrm{NNMHV}}$, Eq. (A.9), one also obtains

$\mathcal{P}_{n+1}^{\mathrm{NNMHV}}(1, \ldots, \hat{n}, \hat{s})=\mathcal{P}_{n}^{\mathrm{NNMHV}}(\hat{1}, \ldots, \hat{n})+\mathcal{O}(\epsilon)$.

Using the same method, we can show that the similar conclusion holds for all $\mathrm{N}^{k} \mathrm{MHV}$ sectors of $\mathcal{N}=4 \mathrm{SYM}$, i.e.,

$\mathcal{P}_{n+1}(1, \ldots, n, s)=\mathcal{P}_{n}(1, \ldots, n)+\mathcal{O}(\epsilon)$.

Let us notice that the function $\mathcal{P}_{n+1}$ is independent of Grassmann variables $\eta_{s}^{A}$ with a certain gauge choice. Using
Eq. (A.26) and (A.13), it is easy to obtain the leading soft gluon divergence,

$$
\begin{gathered}
\mathscr{A}_{n+1}\left(\ldots, g_{s}^{+}\right) \equiv \mathscr{A}_{n+1}^{\mathrm{MHV}}\left(\ldots, g_{s}^{+}\right) \mathcal{P}_{n+1} \\
=\frac{1}{\epsilon^{2}} \frac{\langle n, 1\rangle}{\langle s, 1\rangle\langle n, s\rangle} \mathscr{A}_{n}^{\mathrm{MHV}}\left(\mathcal{P}_{n}+\mathcal{O}(\epsilon)\right) .
\end{gathered}
$$

in the holomorphic soft limit $\lambda_{s} \rightarrow \epsilon \lambda_{s}$. As was discussed earlier, there is no sub-leading soft gluon divergence and here we no longer discuss it. For soft luino, by using Eqs. (A.26) and (A.14), one gets

$$
\begin{aligned}
& \mathscr{A}_{n+1}\left(\ldots, \psi_{s A}\right)=\frac{1}{\epsilon} \frac{\langle n, 1\rangle}{\langle n, s\rangle\langle s, 1\rangle}\left(\frac{\langle n, s\rangle}{\langle n, 1\rangle} \frac{\partial}{\partial \eta_{1}^{A}}+\frac{\langle 1, s\rangle}{\langle 1, n\rangle} \frac{\partial}{\partial \eta_{n}^{A}}\right) \\
& \times \mathscr{A}_{n}^{\operatorname{MHV}}\left(\mathcal{P}_{n}+\mathcal{O}(\epsilon)\right) .
\end{aligned}
$$

in the holomorphic soft limit $\lambda_{s} \rightarrow \epsilon \lambda_{s}$. Both $\mathscr{A}_{n+1}^{\mathrm{MHV}}$ and $\mathcal{P}_{n+1}$ have no singular term in the holomorphic soft limit $\lambda_{s} \rightarrow \epsilon \lambda_{s}$ of a scalar. This implies that there exists no singularity when a external scalar becomes soft in an on-shell amplitude.

\section{Appendix B: Sub-sub-leading soft operator in $\mathcal{N}=8$ SUGRA}

In this appendix, we compute the sub-sub-leading soft operator in $\mathcal{N}=8$ SUGRA by a Taylor expansion in detail.

As shown in Sect. 4, in the holomorphic soft limit $\lambda_{s} \rightarrow$ $\epsilon \lambda_{s}$, the superamplitude $\mathscr{M}_{n+1}$ becomes

$$
\begin{aligned}
& \mathscr{M}_{n+1}(\epsilon)=\frac{1}{\epsilon^{3}} \sum_{a=1}^{n-1} \frac{[s, a]\langle n, a\rangle^{2}}{\langle s, a\rangle\langle n, s\rangle^{2}} \\
& \quad \times \mathscr{M}_{n}\left(\ldots,\left\{\lambda_{a}, \tilde{\lambda}_{a}+\epsilon \frac{\langle n, s\rangle}{\langle n, a\rangle} \tilde{\lambda}_{s}, \eta_{a}+\epsilon \frac{\langle n, s\rangle}{\langle n, a\rangle} \eta_{s}\right\}, \ldots,\right. \\
& \left.\left\{\lambda_{n}, \tilde{\lambda}_{n}+\epsilon \frac{\langle s, a\rangle}{\langle n, a\rangle} \tilde{\lambda}_{s}, \eta_{n}+\epsilon \frac{\langle s, a\rangle}{\langle n, a\rangle} \eta_{s}\right\}\right) .
\end{aligned}
$$

Let us denote

$$
\begin{gathered}
G(\epsilon)=\mathscr{M}_{n}\left(\ldots,\left\{\lambda_{a}, \tilde{\lambda}_{a}+\epsilon \frac{\langle n, s\rangle}{\langle n, a\rangle} \tilde{\lambda}_{s}, \eta_{a}+\epsilon \frac{\langle n, s\rangle}{\langle n, a\rangle} \eta_{s}\right\}\right. \\
\left.\ldots,\left\{\lambda_{n}, \tilde{\lambda}_{n}+\epsilon \frac{\langle s, a\rangle}{\langle n, a\rangle} \tilde{\lambda}_{s}, \eta_{n}+\epsilon \frac{\langle s, a\rangle}{\langle n, a\rangle} \eta_{s}\right\}\right)
\end{gathered}
$$

then expand it in infinitesimal soft parameter $\epsilon$ :

$G(\epsilon)=G(0)+\epsilon G^{\prime}(0)+\frac{1}{2} \epsilon^{2} G^{\prime \prime}(0)+\mathcal{O}\left(\epsilon^{3}\right)$. 
The first two orders contribute to the leading and the subleading soft operators respectively. The second order in $\epsilon$ is

$$
\begin{aligned}
& G^{\prime \prime}(0)=G_{\lambda}^{\prime \prime}(0)+G_{\lambda \eta}^{\prime \prime}(0)+G_{\eta}^{\prime \prime}(0), \\
& G_{\lambda}^{\prime \prime}(0)= \tilde{\lambda}_{s \dot{\alpha}} \tilde{\lambda}_{s \dot{\beta}}\left(\frac{\langle n, s\rangle^{2}}{\langle a, n\rangle^{2}} \frac{\partial^{2}}{\partial \tilde{\lambda}_{a \dot{\alpha}} \partial \tilde{\lambda}_{a \dot{\beta}}}\right. \\
&-2 \frac{\langle n, s\rangle\langle a, s\rangle}{\langle n, a\rangle^{2}} \frac{\partial^{2}}{\partial \tilde{\lambda}_{a \dot{\alpha}} \partial \tilde{\lambda}_{n \dot{\beta}}} \\
&\left.+\frac{\langle a, s\rangle^{2}}{\langle a, n\rangle^{2}} \frac{\partial^{2}}{\partial \tilde{\lambda}_{n \dot{\alpha}} \partial \tilde{\lambda}_{n \dot{\beta}}}\right) \mathscr{M}_{n}, \\
& G_{\lambda_{\eta}}^{\prime \prime}(0)= 2 \tilde{\lambda}_{s \dot{\alpha}} \eta_{s}^{A}\left(\frac{\langle n, s\rangle^{2}}{\langle a, n\rangle^{2}} \frac{\partial^{2}}{\partial \tilde{\lambda}_{a \dot{\alpha}} \partial \eta_{a}^{A}}\right. \\
&+\frac{\langle n, s\rangle\langle s, a\rangle}{\langle a, n\rangle^{2}}\left(\frac{\partial^{2}}{\partial \tilde{\lambda}_{a \dot{\alpha}} \partial \eta_{n}^{A}}+\frac{\partial^{2}}{\partial \tilde{\lambda}_{n \dot{\alpha}} \partial \eta_{a}^{A}}\right) \\
&\left.+\frac{\langle s, a\rangle^{2}}{\langle n, a\rangle^{2}} \frac{\partial^{2}}{\partial \tilde{\lambda}_{n \dot{\alpha}} \partial \eta_{n}^{A}}\right) \mathscr{M}_{n}, \\
& G_{\eta}^{\prime \prime}(0)= \eta_{s}^{A} \eta_{s}^{B}\left(\frac{\langle n, s\rangle^{2}}{\langle a, n\rangle^{2}} \frac{\partial^{2}}{\partial \eta_{a}^{B} \partial \eta_{a}^{A}}\right. \\
&\left.-2 \frac{\langle n, s\rangle\langle a, s\rangle}{\langle n, a\rangle^{2}} \frac{\partial^{2}}{\partial \eta_{a}^{B} \partial \eta_{n}^{A}}+\frac{\langle a, s\rangle^{2}}{\langle a, n\rangle^{2}} \frac{\partial^{2}}{\partial \eta_{n}^{B} \partial \eta_{n}^{A}}\right) \mathscr{M}_{n} .
\end{aligned}
$$

It is important to notice that here the derivative with respect to the Grassmann variable $\eta^{A}$ is left derivative. Plugging the second derivative $G^{\prime \prime}(0)$ on the right hand side of Eq. (B.1), we obtain

$$
\begin{aligned}
& \mathcal{S}^{(2)} \mathcal{M}_{n} \\
& \quad=\frac{1}{2 \epsilon} \sum_{a=1}^{n-1} \frac{[s, a]\langle n, a\rangle^{2}}{\langle s, a\rangle\langle n, s\rangle^{2}}\left(G_{\lambda}^{\prime \prime}(0)+G_{\lambda \eta}^{\prime \prime}(0)+G_{\eta}^{\prime \prime}(0)\right) .
\end{aligned}
$$

The first term (B.5) on the right hand side of Eq. (B.8) has obtained in Cachazo-Strominger's paper [32]:

$S^{(2)}=\frac{1}{2} \sum_{a=1}^{n} \frac{[s, a]}{\langle s, a\rangle} \tilde{\lambda}_{s \dot{\alpha}} \tilde{\lambda}_{s \dot{\beta}} \frac{\partial^{2}}{\partial \tilde{\lambda}_{a \dot{\alpha}} \partial \tilde{\lambda}_{a \dot{\beta}}}$.

By using similar derivation, it is easy to write down the third term (B.7) on the right hand side of Eq. (B.8):

$\mathcal{S}_{\eta}^{(2)}=\frac{1}{2} \sum_{a=1}^{n} \frac{[s, a]}{\langle s, a\rangle} \eta_{s}^{A} \eta_{s}^{B} \frac{\partial^{2}}{\partial \eta_{a}^{B} \partial \eta_{a}^{A}}$.

The second term (B.6) on the right hand side of Eq. (B.8) is given by

$\mathcal{S}_{\lambda \eta}^{(2)} \mathscr{M}_{n}$

$=\sum_{a=1}^{n-1} \tilde{\lambda}_{s \dot{\alpha}} \eta_{s}^{A}\left(\frac{[s, a]}{\langle s, a\rangle} \frac{\partial^{2}}{\partial \tilde{\lambda}_{a \dot{\alpha}} \partial \eta_{a}^{A}}\right.$

$$
\begin{aligned}
& +\frac{[s, a]}{\langle n, s\rangle}\left(\frac{\partial^{2}}{\partial \tilde{\lambda}_{a \dot{\alpha}} \partial \eta_{n}^{A}}+\frac{\partial^{2}}{\partial \tilde{\lambda}_{n \dot{\alpha}} \partial \eta_{a}^{A}}\right) \\
& \left.+\frac{\langle s, a\rangle[s, a]}{\langle n, s\rangle^{2}} \frac{\partial^{2}}{\partial \tilde{\lambda}_{n \dot{\alpha}} \partial \eta_{n}^{A}}\right) \mathscr{M}_{n} .
\end{aligned}
$$

Noticing that

$$
\begin{aligned}
\sum_{a=1}^{n-1}\langle s, a\rangle[s, a] & =\sum_{a=1}^{n-1} 2 k_{s} \cdot k_{a} \\
& =-2 k_{s} \cdot\left(k_{n}+k_{s}\right)=-2 k_{s} \cdot k_{n} \\
& =-\langle s, n\rangle[s, n],
\end{aligned}
$$

then the last term of Eq. (B.11) becomes

$$
\begin{aligned}
& \sum_{a=1}^{n-1} \frac{\langle s, a\rangle[s, a]}{\langle n, s\rangle^{2}} \tilde{\lambda}_{s \dot{\alpha}} \eta_{s}^{A} \frac{\partial^{2}}{\partial \tilde{\lambda}_{n \dot{\alpha}} \partial \eta_{n}^{A}} \mathscr{M}_{n} \\
& =-\frac{[s, n]}{\langle s, n\rangle} \tilde{\lambda}_{s \dot{\alpha}} \eta_{s}^{A} \frac{\partial^{2}}{\partial \tilde{\lambda}_{n \dot{\alpha}} \partial \eta_{n}^{A}} \mathscr{M}_{n} .
\end{aligned}
$$

Write the second term of Eq. (B.11) as follows:

$$
\begin{aligned}
& \sum_{a=1}^{n-1} \frac{[s, a]}{\langle n, s\rangle} \tilde{\lambda}_{s \dot{\alpha}} \eta_{s}^{A} \frac{\partial^{2}}{\partial \tilde{\lambda}_{a \dot{\alpha}} \partial \eta_{n}^{A}} \mathscr{M}_{n} \\
& \quad=\frac{\eta_{s}^{A} \tilde{\lambda}_{s \dot{\beta}} \tilde{\lambda}_{s \dot{\alpha}}}{\langle n, s\rangle} \sum_{a=1}^{n-1} \tilde{\lambda}_{a}^{\dot{\alpha}} \frac{\partial^{2}}{\partial \tilde{\lambda}_{a \dot{\beta}} \partial \eta_{n}^{A}} \mathscr{M}_{n} .
\end{aligned}
$$

Using the global angular momentum conservation, we get

$$
\begin{aligned}
& \tilde{\lambda}_{s \dot{\beta}} \tilde{\lambda}_{s \dot{\alpha}} \sum_{a=1}^{n-1} \tilde{\lambda}_{a}^{\dot{\alpha}} \frac{\partial}{\partial \tilde{\lambda}_{a \dot{\beta}}} \mathscr{M}_{n} \\
& \quad=-\tilde{\lambda}_{s \dot{\beta}} \tilde{\lambda}_{s \dot{\alpha}} \tilde{\lambda}_{n}^{\dot{\alpha}} \frac{\partial}{\partial \tilde{\lambda}_{n \dot{\beta}}} \mathscr{M}_{n}=-[s, n] \tilde{\lambda}_{s \dot{\alpha}} \frac{\partial}{\partial \tilde{\lambda}_{n \dot{\alpha}}} \mathscr{M}_{n} .
\end{aligned}
$$

Thus we have

$$
\begin{aligned}
& \sum_{a=1}^{n-1} \frac{[s, a]}{\langle n, s\rangle} \tilde{\lambda}_{s \dot{\alpha}} \eta_{s}^{A} \frac{\partial^{2}}{\partial \tilde{\lambda}_{a \dot{\alpha}} \partial \eta_{n}^{A}} \mathscr{M}_{n} \\
& \quad=\frac{[s, n]}{\langle s, n\rangle} \tilde{\lambda}_{s \dot{\alpha}} \eta_{s}^{A} \frac{\partial^{2}}{\partial \tilde{\lambda}_{n \dot{\alpha}} \partial \eta_{n}^{A}} \mathscr{M}_{n} .
\end{aligned}
$$

The third term of Eq. (B.11) may be written as:

$$
\begin{aligned}
& \sum_{a=1}^{n-1} \frac{[s, a]}{\langle n, s\rangle} \tilde{\lambda}_{s \dot{\beta}} \eta_{s}^{A} \frac{\partial^{2}}{\partial \tilde{\lambda}_{n \dot{\beta}} \partial \eta_{a}^{A}} \mathscr{M}_{n} \\
& \quad=\frac{\eta_{s}^{A} \tilde{\lambda}_{s} \tilde{\lambda}_{s \dot{\alpha}}}{\langle n, s\rangle} \sum_{a=1}^{n-1} \tilde{\lambda}_{a}^{\dot{\alpha}} \frac{\partial^{2}}{\partial \eta_{a}^{A} \partial \tilde{\lambda}_{n \dot{\beta}}} \mathscr{M}_{n} .
\end{aligned}
$$


Using the supersymmetry, ${ }^{8}$ we have

$$
\begin{aligned}
\tilde{\lambda}_{s} \dot{\alpha} & \sum_{a=1}^{n-1} \tilde{\lambda}_{a}^{\dot{\alpha}} \frac{\partial}{\partial \eta_{a}^{A}} \mathscr{M}_{n} \\
& =-\tilde{\lambda}_{s} \tilde{\alpha}^{\dot{\alpha}} \frac{\partial}{\partial \eta_{n}^{A}} \mathscr{M}_{n}=-[s, n] \frac{\partial}{\partial \eta_{n}^{A}} \mathscr{M}_{n} .
\end{aligned}
$$

Thus we obtain the third term of Eq. (B.11):

$$
\begin{aligned}
& \sum_{a=1}^{n-1} \frac{[s, a]}{\langle n, s\rangle} \tilde{\lambda}_{s \dot{\beta}} \eta_{s}^{A} \frac{\partial^{2}}{\partial \tilde{\lambda}_{n \dot{\beta}} \partial \eta_{a}^{A}} \mathscr{M}_{n} \\
& \quad=\frac{[s, n]}{\langle s, n\rangle} \tilde{\lambda}_{s \dot{\alpha}} \eta_{s}^{A} \frac{\partial^{2}}{\partial \tilde{\lambda}_{n \dot{\alpha}} \partial \eta_{n}^{A}} \mathscr{M}_{n} .
\end{aligned}
$$

Combining all the contributions, we get

$$
\mathcal{S}_{\lambda \eta}^{(2)} \mathscr{M}_{n}=\sum_{a=1}^{n} \frac{[s, a]}{\langle s, a\rangle} \tilde{\lambda}_{s \dot{\alpha}} \eta_{s}^{A} \frac{\partial^{2}}{\partial \tilde{\lambda}_{a \dot{\alpha}} \partial \eta_{a}^{A}} \mathscr{M}_{n}
$$

Finally, we obtain a complete sub-sub-leading soft operator in $\mathcal{N}=8$ SUGRA

$$
\begin{aligned}
\mathcal{S}^{(2)} & =\frac{1}{2} \sum_{a=1}^{n} \frac{[s, a]}{\langle s, a\rangle} \\
& \times\left(\tilde{\lambda}_{s \dot{\alpha}} \tilde{\lambda}_{s \dot{\beta}} \frac{\partial^{2}}{\partial \tilde{\lambda}_{a \dot{\alpha}} \partial \tilde{\lambda}_{a \dot{\beta}}}+2 \tilde{\lambda}_{s \dot{\alpha}} \eta_{s}^{A} \frac{\partial^{2}}{\partial \tilde{\lambda}_{a \dot{\alpha}} \partial \eta_{a}^{A}}+\eta_{s}^{A} \eta_{s}^{B} \frac{\partial^{2}}{\partial \eta_{a}^{B} \partial \eta_{a}^{A}}\right) .
\end{aligned}
$$

\section{References}

1. E. Casali, Soft sub-leading divergences in Yang-Mills amplitudes. JHEP 1408, 077 (2014). arXiv: 1404.5551

2. F. Cachazo, E.Y. Yuan, Are Soft Theorems Renormalized? arXiv: 1405.3413

3. S. He, Y.-T. Huang, C. Wen, Loop Corrections to Soft Theorems in Gauge Theories and Gravity. JHEP 1412, 115 (2014). arXiv: 1405.1410

4. M. Bianchi, S. He, Y.-T. Huang, C. Wen, More on Soft Theorems: Trees, Loops and Strings, arXiv: 1406.5155

5. Z. Bern, S. Davies, J. Nohle, On loop corrections to subleading soft behavior of gluons and gravitons. Phys. Rev. D 90, 085015 (2014). arXiv:1405.1015

6. Z. Bern, S. Davies, P. Di Vecchia, J. Nohle, Low-energy behavior of gluons and gravitons from gauge invariance. Phys. Rev. D 90, 084035 (2014). arXiv:1406.6987

\footnotetext{
8 The SUSY requires that

$\sum_{a=1}^{n} \lambda_{a}^{\alpha} \eta_{a}^{A} \mathscr{M}_{n}=\sum_{a=1}^{n} \tilde{\lambda}_{a}^{\dot{\alpha}} \frac{\partial}{\partial \eta_{a}^{A}} \mathscr{M}_{n}=0$
}

for $n$-point on-shell superamplitudes.
7. J. Broedel, M. de Leeuw, J. Plefka, M. Rosso, Constraining subleading soft gluon and graviton theorems. Phys. Rev. D 90, 065024 (2014). arXiv:1406.6574

8. C.D. White, Diagrammatic insights into next-to-soft corrections. Phys. Lett. B 737, 216 (2014). arXiv: 1406.7184

9. B.U.W. Schwab, A. Volovich, Subleading soft theorem in arbitrary dimension from scattering equations. Phys. Rev. Lett. 113, 101601 (2014). arXiv:1404.7749

10. N. Afkhami-Jeddi, Soft Graviton Theorem in Arbitrary Dimensions, arXiv: 1405.3533

11. M. Zlotnikov, Sub-sub-leading soft-graviton theorem in arbitrary dimension. JHEP 1410, 148 (2014). arXiv:1407.5936

12. C. Kalousios, F. Rojas, Next to subleading soft-graviton theorem in arbitrary dimensions. JHEP 1501, 107 (2015). arXiv: 1407.5982

13. S.G. Naculich, Scattering equations and BCJ relations for gauge and gravitational amplitudes with massive scalar particles. JHEP 1409, 029 (2014). arXiv:1407.7836

14. B.U.W. Schwab, Subleading soft factor for string disk amplitudes. JHEP 1408, 062 (2014). arXiv: 1406.4172

15. Y. Geyer, A.E. Lipstein, L. Mason, Ambitwistor strings at null infinity and subleading soft limits. Class. Quant. Grav. 32, 055003 (2015). arXiv:1406.1462

16. A.J. Larkoski, Conformal invariance of the subleading soft theorem in gauge theory. Phys. Rev. D 90, 087701 (2014). arXiv:1405.2346

17. V. Lysov, S. Pasterski, A. Strominger, Low's subleading soft theorem as a symmetry of QED. Phys. Rev. Lett. 113, 111601 (2014). arXiv: 1407.3814

18. T. He, P. Mitra, A.P. Porfyriadis, A. Strominger, New symmetries of massless QED. JHEP 1410, 112 (2014). arXiv: 1407.3789

19. Y.-J. Du, B. Feng, C.-H. Fu, Y. Wang, Note on soft graviton theorem by KLT Relation. JHEP 1411, 090 (2014). arXiv:1408.4179

20. C.D. White, Factorization properties of soft graviton amplitudes. JHEP 1105, 060 (2011). arXiv:1103.2981

21. E. Laenen, G. Stavenga, C.D. White, Path integral approach to eikonal and next-to-eikonal exponentiation. JHEP 0903, 054 (2009). arXiv:0811.2067

22. E. Laenen, L. Magnea, G. Stavenga, C.D. White, Next-to-eikonal corrections to soft gluon radiation: a diagrammatic approach. JHEP 1101, 141 (2011). arXiv: 1010.1860

23. S. Oxburgh, C.D. White, BCJ duality and the double copy in the soft limit. JHEP 1302, 127 (2013). arXiv: 1210.1110

24. T. Adamo, E. Casali, D. Skinner, Perturbative gravity at null infinity. Class. Quant. Grav. 31, 225008 (2014). arXiv:1405.5122

25. A. Strominger, On BMS invariance of gravitational scattering. JHEP 1407, 152 (2014). arXiv:1312.2229

26. T. He, V. Lysov, P. Mitra and A. Strominger, BMS supertranslations and Weinberg's soft graviton theorem, arXiv:1401.7026

27. D. Kapec, V. Lysov, S. Pasterski, A. Strominger, Semiclassical Virasoro symmetry of the quantum gravity $\mathcal{S}$-Matrix. JHEP 1408, 058 (2014). arXiv: 1406.3312

28. S. Weinberg, Infrared photons and gravitons. Phys. Rev. B 140, $516(1965)$

29. F.E. Low, Scattering of light of very low frequency by systems of spin 1/2. Phys. Rev. 96, 1428 (1954)

30. F.E. Low, Bremsstrahlung of very low-energy quanta in elementary particle collisions. Phys. Rev. 110, 974 (1958)

31. D.J. Gross, R. Jackiw, Low-energy theorem for graviton scattering. Phys. Rev. 166, 1287 (1968)

32. F. Cachazo, A. Strominger, Evidence for a New Soft Graviton Theorem, arXiv: 1404.4091

33. N. Arkani-Hamed, J. Kaplan, On tree amplitudes in gauge theory and gravity. JHEP 0804, 076 (2008). arXiv:0801.2385

34. R. Britto, F. Cachazo, B. Feng, New recursion relations for tree amplitudes of gluons, Nucl. Phys. B 715, 499 (2005). hep-th/0412308 
35. R. Britto, F. Cachazo, B. Feng, E. Witten, Direct proof of tree-level recursion relation in Yang-Mills theory. Phys. Rev. Lett. 94, 181602 (2005). hep-th/0501052

36. F. Cachazo, P. Svrcek, Tree Level Recursion Relations in General Relativity, hep-th/0502160

37. P. Benincasa, C. Boucher-Veronneau, F. Cachazo, Taming tree amplitudes in general relativity. JHEP 0711, 057 (2007). hep-th/0702032

38. J. Bedford, A. Brandhuber, B.J. Spence, G. Travaglini, A recursion relation for gravity amplitudes. Nucl. Phys. B 721, 98 (2005). hep-th/0502146

39. L.J. Dixon, Calculating scattering amplitudes efficiently, hep-ph/9601359

40. E. Witten, Perturbative gauge theory as a string theory in twistor space. Commun. Math. Phys. 252, 189 (2004). hep-th/0312171

41. Z. Xu, D.-H. Zhang, L. Chang, Helicity amplitudes for multiple bremsstrahlung in massless nonabelian gauge theories. Nucl. Phys. B 291, 392 (1987)

42. F. Cachazo, S. He, E.Y. Yuan, Scattering of massless particles in arbitrary dimension. Phys. Rev. Lett. 113, 171601 (2014). arXiv: 1307.2199

43. F. Cachazo, S. He, E.Y. Yuan, Scattering of massless particles: scalars, gluons and gravitons. JHEP 1407, 033 (2014). arXiv: 1309.0885

44. F. Cachazo, S. He, E.Y. Yuan, Scattering equations and KLT orthogonality. Phys. Rev. D 90, 065001 (2014). arXiv:1306.6575

45. F. Cachazo, S. He, E.Y. Yuan, Scattering in three dimensions from rational maps. JHEP 1310, 141 (2013). arXiv:1306.2962

46. J.M. Drummond, J.M. Henn, J. Plefka, Yangian symmetry of scattering amplitudes in $\mathcal{N}=4$ super Yang-Mills theory. JHEP 0905, 046 (2009). arXiv:0902.2987

47. J.M. Drummond, J. Henn, G.P. Korchemsky, E. Sokatchev, Dual superconformal symmetry of scattering amplitudes in $\mathcal{N}=$ 4 super-Yang-Mills theory. Nucl. Phys. B 828, 317 (2010). arXiv:0807.1095

48. A. Brandhuber, P. Heslop, G. Travaglini, A Note on dual superconformal symmetry of the $\mathcal{N}=4$ super Yang-Mills S-matrix. Phys. Rev. D 78, 125005 (2008). arXiv:0807.4097

49. G.P. Korchemsky, E. Sokatchev, Symmetries and analytic properties of scattering amplitudes in $\mathcal{N}=4$ SYM theory. Nucl. Phys. B 832, 1 (2010). arXiv:0906.1737

50. G.P. Korchemsky, E. Sokatchev, Superconformal invariants for scattering amplitudes in $\mathcal{N}=4$ SYM theory. Nucl. Phys. B 839, 377 (2010). arXiv:1002.4625

51. N. Beisert et al., Review of AdS/CFT integrability: an overview. Lett. Math. Phys. 99, 3 (2012). arXiv:1012.3982

52. Z. Bern, J.J.M. Carrasco, H. Johansson, New relations for gauge-theory amplitudes. Phys. Rev. D 78, 085011 (2008). arXiv:0805.3993

53. L.J. Dixon, Scattering amplitudes: the most perfect microscopic structures in the universe. J. Phys. A 44, 454001 (2011). arXiv:1105.0771

54. H. Elvang, Y.-T. Huang, Scattering amplitudes, arXiv:1308.1697

55. J. Henn, J.C. Plefka, Scattering amplitudes in gauge theories. In: Lecture Notes in Physics, vol. 883, Springer, Berlin (2014)

56. N. Arkani-Hamed, F. Cachazo, J. Kaplan, What is the simplest quantum field theory? JHEP 1009, 016 (2010). arXiv:0808.1446

57. J.M. Drummond, J.M. Henn, All tree-level amplitudes in $\mathcal{N}=4$ SYM. JHEP 0904, 018 (2009). arXiv:0808.2475

58. M. Bianchi, H. Elvang, D.Z. Freedman, Generating tree amplitudes in $\mathcal{N}=4$ SYM and $\mathcal{N}=8$ SG. JHEP 0809, 063 (2008). arXiv:0805.0757

59. H. Elvang, D.Z. Freedman, M. Kiermaier, Solution to the ward identities for superamplitudes. JHEP 1010, 103 (2010). arXiv:0911.3169
60. J.M. Drummond, M. Spradlin, A. Volovich, C. Wen, Tree-level amplitudes in $\mathcal{N}=8$ supergravity. Phys. Rev. D 79, 105018 (2009). arXiv:0901.2363

61. L.J. Dixon, J.M. Henn, J. Plefka, T. Schuster, All tree-level amplitudes in massless QCD, JHEP 1101035 (2011) . arXiv:1010.3991

62. R. Kallosh, C.H. Lee, T. Rube, $\mathcal{N}=8$ Supergravity 4-point amplitudes. JHEP 0902, 050 (2009). arXiv:0811.3417

63. D. Nandan, C. Wen, Generating all tree amplitudes in $\mathcal{N}=4 \mathrm{SYM}$ by inverse soft limit. JHEP 1208, 040 (2012). arXiv:1204.4841

64. C. Boucher-Veronneau, A.J. Larkoski, Constructing amplitudes from their soft limits. JHEP 1109, 130 (2011). arXiv:1108.5385

65. R. Britto, B. Feng, R. Roiban, M. Spradlin, A. Volovich, All split helicity tree-level gluon amplitudes. Phys. Rev. D 71, 105017 (2005). hep-th/0503198

66. N.E.J. Bjerrum-Bohr, O.T. Engelund, Gravitino interactions from Yang-Mills theory. Phys. Rev. D 81, 105009 (2010). arXiv: 1002.2279

67. M.T. Grisaru, H.N. Pendleton, Some properties of scattering amplitudes in supersymmetric theories. Nucl. Phys. B 124, 81 (1977)

68. M.T. Grisaru, H.N. Pendleton, P. van Nieuwenhuizen, Supergravity and the S Matrix. Phys. Rev. D 15, 996 (1977)

69. T. Melia, Getting more flavour out of one-flavour QCD. Phys. Rev. D 89, 074012 (2014). arXiv:1312.0599

70. T. Melia, Dyck words and multiquark primitive amplitudes. Phys. Rev. D 88, 014020 (2013). arXiv: 1304.7809

71. F.A. Berends, W.T. Giele, Multiple soft gluon radiation in parton processes. Nucl. Phys. B 313, 595 (1989)

72. H. Kawai, D.C. Lewellen, S.H.H. Tye, A relation between tree amplitudes of closed and open strings. Nucl. Phys. B 269, 1 (1986)

73. Z. Bern, Perturbative quantum gravity and its relation to gauge theory, Living Rev. Rel. 55 (2002). gr-qc/0206071

74. Z. Bern, A.K. Grant, Perturbative gravity from QCD amplitudes. Phys. Lett. B 457, 23 (1999). hep-th/9904026

75. F.A. Berends, W.T. Giele, H. Kuijf, On relations between multigluon and multigraviton scattering. Phys. Lett. B 211, 91 (1988)

76. N.E.J. Bjerrum-Bohr, P.H. Damgaard, T. Sondergaard, P. Vanhove, The momentum kernel of gauge and gravity theories. JHEP 1101, 001 (2011). arXiv:1010.3933

77. N.E.J. Bjerrum-Bohr, P.H. Damgaard, B. Feng, T. Sondergaard, Gravity and Yang-Mills amplitude relations. Phys. Rev. D 82, 107702 (2010). arXiv: 1005.4367

78. N.E.J. Bjerrum-Bohr, P.H. Damgaard, B. Feng, T. Sondergaard, Proof of gravity and Yang-Mills amplitude relations. JHEP 1009, 067 (2010). arXiv:1007.3111

79. V.P. Nair, A current algebra for some gauge theory amplitudes. Phys. Lett. B 214, 215 (1988)

80. L.J. Dixon, A brief introduction to modern amplitude methods, arXiv: 1310.5353

81. Z. Kunszt, Combined use of the Calkul method and $N=1$ supersymmetry to calculate QCD six parton processes. Nucl. Phys. B 271, 333 (1986)

82. S.J. Bidder, D.C. Dunbar, W.B. Perkins, Supersymmetric Ward identities and NMHV amplitudes involving gluinos. JHEP 0508, 055 (2005). hep-th/0505249

83. M. Luo, C. Wen, Recursion relations for tree amplitudes in super gauge theories. JHEP 0503, 004 (2005). hep-th/0501121

84. M. Luo, C. Wen, Compact formulas for all tree amplitudes of six partons. Phys. Rev. D 71, 091501 (2005). hep-th/0502009

85. R. Kallosh, T. Kugo, The Footprint of $E_{7(7)}$ amplitudes of $\mathcal{N}=8$ supergravity. JHEP 0901, 072 (2009). arXiv:0811.3414

86. S. He, H. Zhu, A note on single soft scalar emission of $\mathcal{N}=8$ SUGRA and $E_{7(7)}$ symmetry. JHEP 1007, 025 (2010). arXiv:0812.4533

87. E. Cremmer, B. Julia, The SO(8) supergravity. Nucl. Phys. B 159, $141(1979)$ 
88. C. Hillmann, Generalized $E_{7(7)}$ coset dynamics and $D=11$ supergravity. JHEP 0903, 135 (2009). arXiv:0901.1581

89. R. Kallosh, $E_{7(7)}$ Symmetry and finiteness of $\mathcal{N}=8$ supergravity. JHEP 1203, 083 (2012). arXiv: 1103.4115

90. R. Kallosh, M. Soroush, Explicit action of $E_{7(7)}$ on $\mathcal{N}=8$ supergravity fields. Nucl. Phys. B 801, 25 (2008). arXiv:0802.4106

91. B. Feng, S. He, KLT and new relations for $\mathcal{N}=8$ SUGRA and $\mathcal{N}=4$ SYM. JHEP 1009, 043 (2010). arXiv:1007.0055
92. Z. Bern, L.J. Dixon, D.C. Dunbar, M. Perelstein, J.S. Rozowsky, On the relationship between Yang-Mills theory and gravity and its implication for ultraviolet divergences. Nucl. Phys. B 530, 401 (1998). hep-th/9802162

93. A. Strominger, Asymptotic symmetries of Yang-Mills theory. JHEP 1407, 151 (2014). arXiv:1308.0589 